\title{
Effectiveness and Safety Profile of Alternative Tobacco and Nicotine Products for Smoking Reduction and Cessation: A Systematic Review
}

\author{
Neily Zakiyah $\mathbb{1 D}^{1,2}$ \\ Febby V Purwadi (D) ${ }^{1,2}$ \\ Widya $N$ Insani $\mathbb{1 D}^{1-3}$ \\ Rizky Abdulah (D) ${ }^{1,2}$ \\ Irma M Puspitasari $\mathbb{1 D}^{1,2}$ \\ Melisa I Barliana $\mathbb{D D}^{1,2,4}$ \\ Ronny Lesmana ${ }^{2,5,6}$ \\ Amaliya Amaliya $\mathbb{B D}^{7}$ \\ Auliya A Suwantika $\mathbb{D}^{1,2,8}$
}

'Department of Pharmacology and Clinical Pharmacy, Faculty of Pharmacy, Universitas Padjadjaran, Bandung, West Java, Indonesia; ${ }^{2}$ Center of Excellence in Higher Education for Pharmaceutical

Care Innovation, Universitas Padjadjaran, Bandung, West Java, Indonesia; ${ }^{3}$ Research Department of Practice and Policy, School of Pharmacy, University College London, London, UK; ${ }^{4}$ Department of Biological Pharmacy, Biotechnology Pharmacy Laboratory, Faculty of Pharmacy, Universitas Padjadjaran, Bandung, West Java, Indonesia; ${ }^{5}$ Division of Physiology, Department of Biomedical Science, Faculty of Medicine, Universitas Padjadjaran, Bandung, West Java, Indonesia; ${ }^{6}$ Division of Biological Activity, Central Laboratory, Universitas Padjadjaran, Bandung, West Java, Indonesia; ' ${ }^{7}$ Department of Periodontics, Faculty of Dentistry, Universitas Padjadjaran, Bandung, West Java, Indonesia; ${ }^{8}$ Center for Health Technology Assessment, Universitas Padjadjaran,

Bandung, West Java, Indonesia

Correspondence: Neily Zakiyah Department of Pharmacology and Clinical Pharmacy, Faculty of Pharmacy, Universitas Padjadjaran, Bandung, West Java, Indonesia

Tel +62-22-77962

Email neily.zakiyah@unpad.ac.id
Background: Alternative tobacco and nicotine products such as electronic cigarettes (EC), smokeless tobacco, and nicotine replacement therapy (NRT) are currently being assessed as options in tobacco harm reduction due to their potential role in smoking reduction and smoking cessation.

Objective: To provide the current evidence on the effectiveness and safety of various alternative tobacco and nicotine products for smoking reduction and cessation.

Methods: A systematic review using databases from MEDLINE (PubMed), EMBASE, and The Cochrane Library was conducted up to December 2020 to identify eligible experimental and observational studies assessing the use of alternative tobacco and nicotine products on smoking reduction and smoking cessation and the safety of these products. The Cochrane Risk of Bias 2 (RoB 2) and ROBINS-I tools were used to assess the risk of bias of the included studies. Results were described through a narrative synthesis of the evidence.

Results: From 1955 retrieved references, 44 studies (31 randomized controlled trials/RCTs and 13 prospective cohort studies) met the inclusion criteria and were included in the review. Twenty-nine studies were assessing EC, one study evaluated heat-not-burn (HNB) product, five studies were focused on snus, and nine studies assessed NRT in the form of nicotine patch, gum, etc. The overall results suggested that alternative tobacco and nicotine products in the form of EC, snus, and NRT can moderately reduce daily cigarette consumption and has potential to assist smoking cessation attempts, with fewer adverse events.

Conclusion: The findings suggested that alternative tobacco and nicotine products have a potential role in assisting smoking reduction and cessation, highlighting their role in the tobacco harm reduction approach. Further studies should focus on investigating long-term outcomes, safety, and effectiveness of alternative tobacco and nicotine products to better inform smoking reduction/cessation policy.

PROSPERO Registration Number: CRD42020205830.

Keywords: cigarette smoking, smoking cessation, smoking reduction, nicotine, e-cigarettes, snus, nicotine replacement therapy, harm reduction

\section{Background}

Smoking is the most significant modifiable risk factor of morbidity and mortality, associated with a wide range of diseases, such as chronic obstructive pulmonary disease (COPD), coronary heart disease (CHD), stroke, lung cancer, and other chronic diseases. $^{1-4}$ The prevalence of COPD, in particular, increased with prolonged smoking duration both in men and women. ${ }^{5}$ Smoking-related illnesses are estimated to cause 
more than eight million deaths annually, worldwide. ${ }^{6}$ Recent population-based studies showed that prolonged smoking was associated with 10 years reduced life expectancy, and cessation before the age of 40 reduced the risk of smokingrelated death by up to $90 \%{ }^{7,8}$ Evidence suggests that approximately half of the smokers reported having made a quit attempt in the past year, but less than $10 \%$ of such attempts succeed. ${ }^{9,10}$

Behavioral approaches such as cognitive behavior therapy, group therapy programs, or individual counseling sessions with or without pharmacotherapy, eg, varenicline, bupropion, etc, have been proven effective for smoking cessation interventions. $^{11,12}$ In patients with COPD, improved lung function and respiratory symptoms were observed in those who received nicotine partial agonist, in addition to individual counseling. ${ }^{13,14}$ However, these interventions have to be delivered by professional facilitators such as physicians, clinical psychologists, nurses, etc. ${ }^{12}$ Alternative tobacco and nicotine products such as nicotine replacement therapy (NRTs), electronic nicotine delivery systems (ENDS), electronic cigarette (e-cigarette/EC), and low-nitrosamine smokeless tobacco, are self-help initiated, less invasive interventions that have the potential to assist cessation by attenuating withdrawal symptoms. ${ }^{15-18}$ These adjunct strategies facilitate a transition to abstinence, typically by providing either a markedly lower level or no nicotine, but with much-reduced health risks as a substantially lower amount of harmful chemical constituents are present. ${ }^{19,20}$ Several clinical trials showed that alternative nicotine and tobacco products were more effective for facilitating sustained abstinence and rated significantly more pleasant than other interventions. ${ }^{21,22}$ These harm reduction attempts have the potential to generate tangible public health benefits; nevertheless, this is counterbalanced with concern regarding widespread re-normalization of addiction behavior and its adverse consequences. ${ }^{23,24}$ In a small-scale laboratorybased study, Vardavas et al showed that adverse pulmonary effect was observed following the use of alternative nicotine products. $^{25}$ Previous studies showed that the alternative tobacco and nicotine products are currently used by $0.5-5 \%$ of the adult population, ${ }^{26-28}$ with up to $20 \%$ of smokers using these products as reduction/cessation aid. ${ }^{29-32}$ However, there is little clarity regarding the overall effectiveness and safety of the alternative tobacco and nicotine products and their role in smoking reduction and cessation. ${ }^{17,33-35}$

Assessing the risks and benefits of the alternative tobacco and nicotine products are challenging due to the lack of accurate biomarkers ${ }^{36,37}$ and paucity of evidence in this regard. ${ }^{38,39}$ A comprehensive understanding of the role of these products is important to better inform tobacco harm reduction policy. Previous systematic reviews have been conducted, but these reviews primarily focused on a single form of alternative tobacco and nicotine products, ${ }^{17,23,34,36,38,40}$ did not investigate the safety, ${ }^{41,42}$ and performed within the context of a single country, limiting its generalizability. ${ }^{43}$ Thus, this comprehensive systematic review was performed to provide an understanding of the overall effectiveness and safety of the alternative tobacco and nicotine products for smoking reduction and/or cessation.

\section{Methods}

This systematic review was conducted in accordance with the Preferred Reporting Items for Systematic Reviews and Meta-Analyses (PRISMA; Supplementary materials). ${ }^{44}$ The objectives and methods of this review were registered in the International Prospective Register of Systematic Review (PROSPERO; Supplementary materials).

\section{Search Strategy}

The following databases were used to identify relevant literature, ie, MEDLINE (PubMed), EMBASE, and The Cochrane Library up to December 2020. A systematic search was conducted in these electronic databases to identify relevant studies on the topic of alternative tobacco and nicotine products. The definition of alternative tobacco and nicotine products in this study included heat-not-burn (HNB) products, electronic cigarette (EC), smokeless tobacco such as chewing tobacco, snuff, and snus, and nicotine replacement therapy (NRT) in the form of gum, transdermal patch, nasal spray, oral inhaler, etc. ${ }^{45-49}$ Details on the definition of alternative tobacco and nicotine products are provided in Supplementary materials Table S1.

The following keywords were used for the search: "Electronic Nicotine Delivery Systems" OR "E-Cigarette Vapor" OR "Vaping” OR “e-cigarette” OR “tobacco, smokeless" OR "Tobacco Use Cessation Devices" OR "tobacco, waterpipe" OR "Smoking Water Pipes" AND "drug-related side effects and adverse reactions" OR "Smoking Cessation" OR "Smoking Reduction" (for details on search strategy in all databases see $\underline{\text { Supplementary materials Table S1). }}$

\section{Study Selection}

The search records from all electronic databases were exported to Mendeley reference manager and checked for duplicates. Screening process was carried out in two 
stages, ie, initial screening based on title and abstracts followed by full-text screening. Both screening processes were independently performed by two reviewers (NZ and FVP). Any discrepancies were resolved by consensus or by discussions with a third and fourth reviewer (AAS and WNI). The following inclusion criteria were used for the screening process: published articles were selected if they assessed the utilization of alternative tobacco and nicotine products in terms of smoking reductions and smoking cessation or assessing the safety profile of alternative tobacco and nicotine products in terms of reported clinicalrelated adverse event or clinical and laboratory-measured adverse events in the adult population, published in the last 10 years. Studies on animals and cells, any protocol articles, conference proceedings, review articles, and nonEnglish studies were excluded in the initial screening process. In the full-text screening, we included both experimental (randomized-controlled trials/RCTs) and observational studies (retrospective/prospective cohort, case-control design and nonintervention arms of randomized controlled trials). Irrelevant studies, crosssectional, case series, and case reports were excluded.

\section{Data Syntheses and Extraction}

From each included study, two reviewers (NZ and FVP) extracted data using a predetermined standardized data extraction form. This form was approved by all authors and amended as required. We extracted data regarding study characteristics (author, year of publication, country, study design, characteristics of participants, number of participants, type of interventions, outcome measure, length of study and funding source), and study design. We also extracted study outcomes in terms of smoking reduction, smoking cessation and adverse events along with its reported effect measures.

\section{Risk of Bias and Quality Assessments}

Risk of bias and quality assessments were independently evaluated by two reviewers (NZ and FVP) using The Cochrane Risk of Bias 2 tool (RoB 2) for RCTs, in which each included study was assessed qualitatively using five domains ie, randomization process, deviations from intended interventions, missing outcome data, measurement of the outcome, and selection of the reported result. ${ }^{50}$ The overall bias for each study was then classified as high, some concerns or low risk of bias, based on the criteria listed in the RoB 2 detailed guideline. ${ }^{51}$ Risk of bias graph was derived from this tool. The ROBINS-I tool was used for assessing the risk of bias in nonrandomized and observational studies. ${ }^{52}$ The following domains were evaluated, ie confounding, selection of participants, classifications of interventions, deviations from intended intervention, missing data, measurement of outcome and selection of reported results. The overall risk of bias from these domains was qualitatively categorized as critical, serious, moderate or low risk of bias, based on the criteria explained in the ROBINS-I detailed guideline. ${ }^{53}$

\section{Results}

\section{Systematic Search}

The initial search retrieved 1955 records in PubMed, Embase and the Cochrane Library. After removing 363 duplicates, 1592 articles were screened by title and abstract, excluding 889 records. After the full text screening on 703 articles, 43 articles were included in the systematic review (Figure 1). One extra relevant study ${ }^{54}$ was identified from the included references, resulting in the final inclusion of 44 studies.

In terms of study design, 31 studies were RCTs (experimental) and the remaining 13 studies were prospective cohort studies (observational). Based on criteria of alternative tobacco and nicotine products explained previously in the methods, 29 included articles were assessing $\mathrm{EC},{ }^{22,55-82}$ in which 16 articles were RCTs, ${ }^{22,55-69}$ and 13 studies were prospective cohort studies, ${ }^{70-82}$ one RCT assessing HNB products, ${ }^{83}$ five RCTs assessing snus (categorized as smokeless tobacco $)^{18,54,84-86}$ and nine RCTs focusing on NRT (eg, nicotine patch, gum, etc) ${ }^{87-95} \mathrm{We}$ did not find any observational studies in categories other than EC products.

\section{Characteristics of Included Studies}

The general characteristics and study outcomes of included studies are provided in Table 1. The majority of the studies were assessing EC (68\%), followed by NRT (21\%), smokeless tobacco (9\%) and HNB products $(2 \%)$. Most of the included studies were carried out in highincome countries, such as the USA (20/43), Italy (7/43) and the UK (5/43). The length of included RCTs varied, from days, weeks, to one year. Details on the outcomes in smoking reduction, smoking cessation and adverse events of the included studies are summarized in Supplementary $\underline{\text { materials. Table } \mathrm{S} 1}$ 


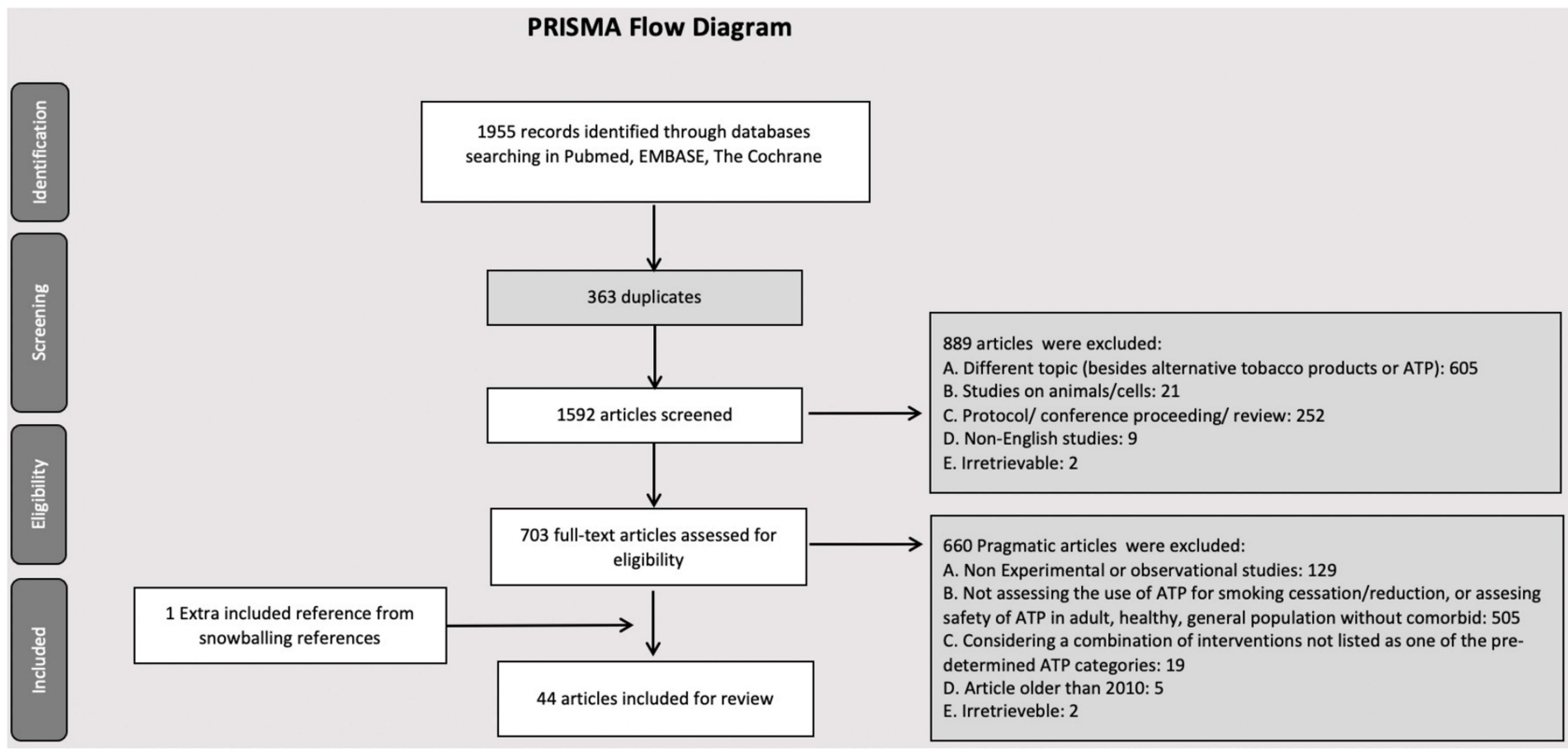

Figure I PRISMA flow diagram of study selection.

Notes:PRISMA figure adapted from Moher D, Liberati A, Tetzlaff J, Altman DG, Group TP. Preferred reporting items for systematic reviews and meta-analyses: the PRISMA statement. PLoS Med. 2009;6(7):el000097. ${ }^{44}$ @ 2009 Moher et al. Creative Commons Attribution License.

\section{Smoking Reduction}

There were nine RCTs $22,55-57,59,63,66,68,69$ and nine prospective cohort studies ${ }^{70-74,78,80-82}$ in the EC group, four RCTs on snus ${ }^{18,54,85,86}$ and two RCTs on $\mathrm{NRT}^{87,91}$ which evaluated the effect of alternative tobacco and nicotine products in smoking reduction. Although variation in number of participants and length of the study was observed between included RCTs in the EC group, all studies reported moderately smoking reduction among trial participants, in which five studies $22,55,57,63,69$ reported approximately $50 \%$ or more reduction in the number of cigarette consumption per day. In addition, three studies reported a greater reduction in nicotine EC compared to nonnicotine EC. ${ }^{63,66,69}$ Results from cohort studies suggested a similar trend, that the use of EC resulted in a considerably decreased number of cigarettes per day, ${ }^{71-}$ $74,80-82$ and that EC use was associated with higher odds of reduced daily cigarette consumption. ${ }^{70,74}$ Nevertheless, contradictory results were also observed among studies, as two studies argued that EC might decrease the likelihood of lowering cigarette consumption. ${ }^{70,78}$

Studies on the use of snus for smoking reduction suggested that snus can lead to a decline in number of cigarettes used per day, although the observed reductions were moderate, approximately in a range of $20-30 \% .{ }^{18,85,86} \mathrm{In}$ addition, one study that defined their primary endpoint as smoking reduction $\geq 50 \%$ suggested that there was no statistically significant difference between snus and the placebo group, although the proportion of participants that reported more than $75 \%$ reduction was significantly higher in the snus group. ${ }^{54}$ Two out of four included RCTs on snus suggested that snus may be inefficient as a harm reduction approach compared to another type of alternative tobacco and nicotine products, ie nicotine gum, ${ }^{86}$ and that provision of snus did not increase smoking abstinence. ${ }^{18}$ Furthermore, that RCTs that focused on NRT in the form of nicotine gum and transdermal nicotine patches also reported a moderate reduction in cigarette consumption. ${ }^{87,91}$

\section{Smoking Cessation}

In terms of smoking cessation, seven included RCTs ${ }^{22,55-}$ $57,59,66,69$ and all included prospective cohort studies ${ }^{70-82}$ assessing EC were observing the quit attempts among participants. Due to variation in the length of the study, the definition of smoking cessation was also varied between studies. All seven RCTs had a long duration of observation, and smoking abstinence was observed in a prolonged manner, ie, continuous abstinence ranging from six months to one year. Overall, the results from seven RCTs in the EC group suggested a very modest portion of subjects who were abstinent from cigarette smoking. ${ }^{22,55-57,59,66,69}$ The results from cohort studies showed conflicting results with regard to smoking cessation. Ten cohort studies indicated that the use of EC was associated 


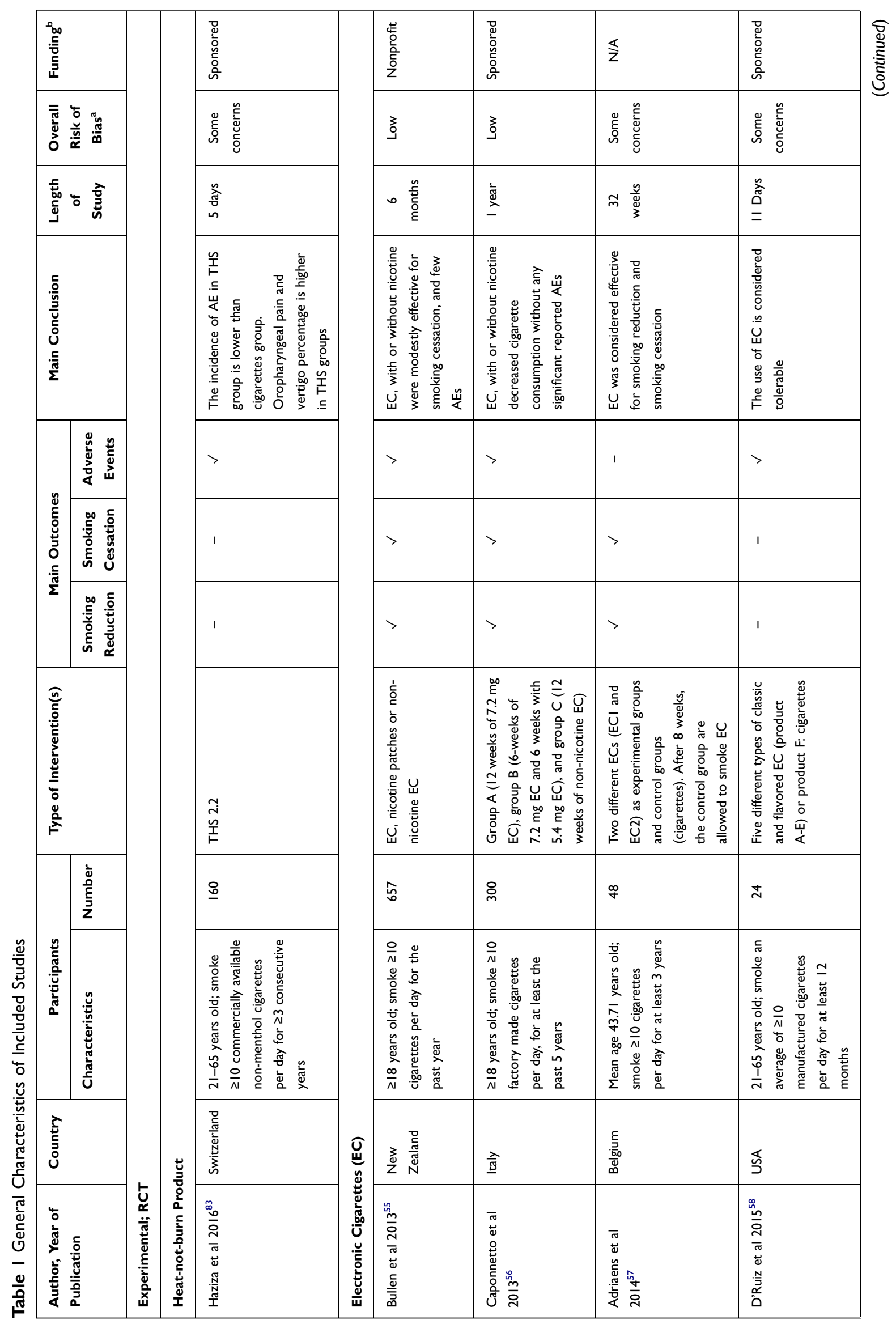




\begin{tabular}{|c|c|c|c|c|c|c|c|}
\hline \multicolumn{2}{|l|}{ 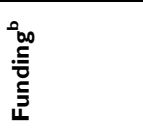 } & 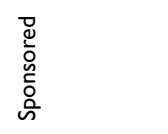 & 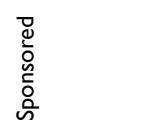 & 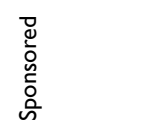 & $\overleftarrow{z}$ & 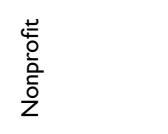 & 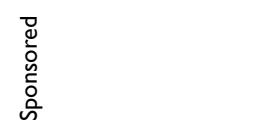 \\
\hline \multicolumn{2}{|c|}{ 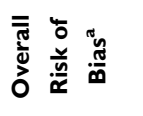 } & 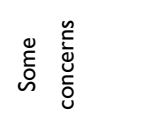 & 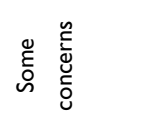 & 3 & 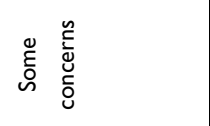 & 3 & 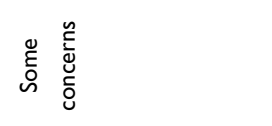 \\
\hline \multicolumn{2}{|c|}{ 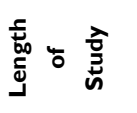 } & 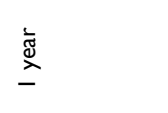 & $\simeq \stackrel{\frac{\tilde{g}}{\omega}}{\xi}$ & 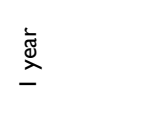 & 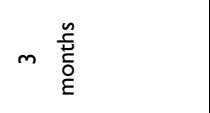 & 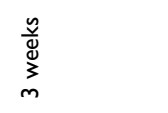 & 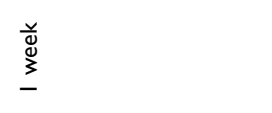 \\
\hline \multicolumn{2}{|c|}{ 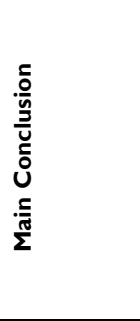 } & 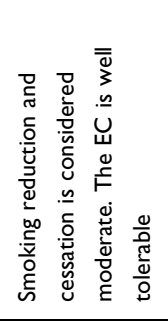 & 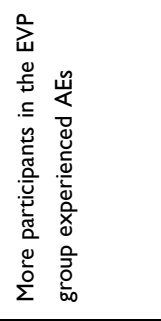 & 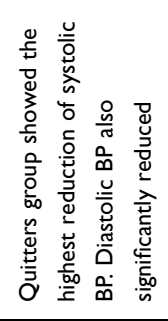 & 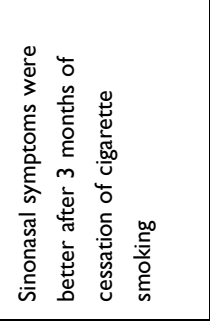 & 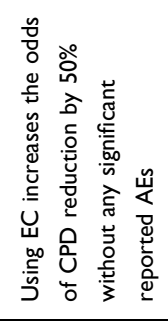 & 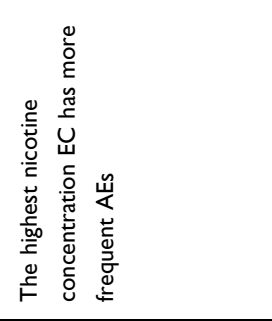 \\
\hline \multirow{3}{*}{ 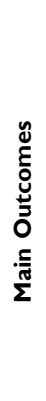 } & 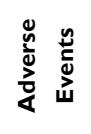 & $>$ & $>$ & $>$ & $>$ & $>$ & $>$ \\
\hline & 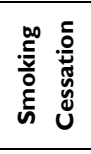 & $>$ & 1 & 1 & 1 & 1 & 1 \\
\hline & 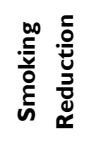 & $>$ & 1 & 1 & 1 & $>$ & 1 \\
\hline \multicolumn{2}{|c|}{ 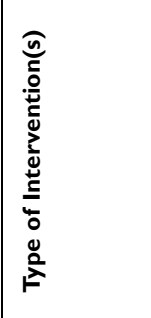 } & 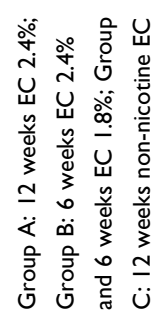 & 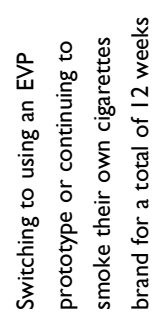 & 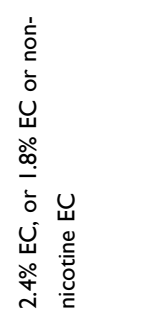 & 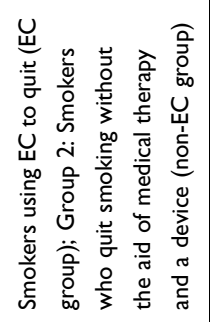 & 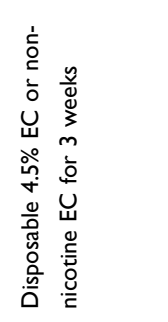 & 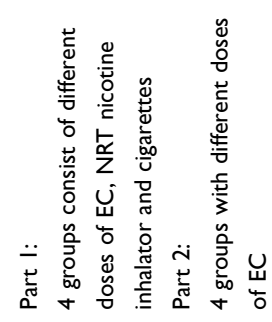 \\
\hline \multirow[b]{2}{*}{ 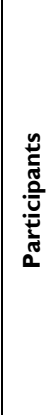 } & $\begin{array}{l}\text { ऐ̀ } \\
\text { है } \\
\bar{z}\end{array}$ & $\underline{\infty}$ & $\stackrel{\mathrm{p}}{\mathrm{m}}$ & ষ্ల & $\stackrel{\infty}{\circ}$ & 。 & $\stackrel{\searrow}{~}$ \\
\hline & 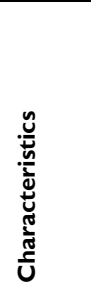 & 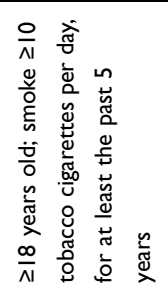 & 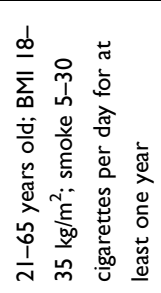 & 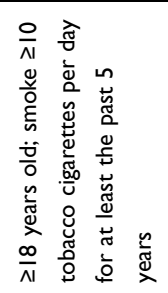 & 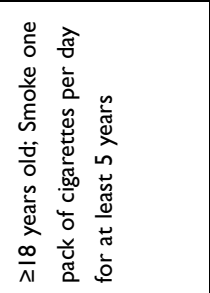 & 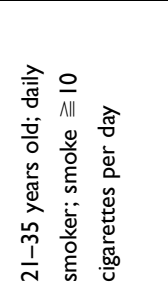 & 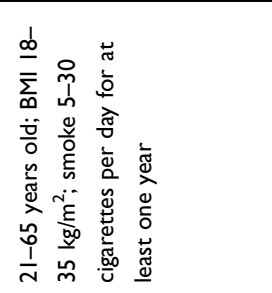 \\
\hline \multicolumn{2}{|l|}{ 竧 } & 첲 & 弚 & 롶 & 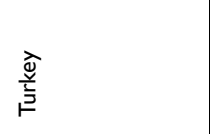 & $\overleftrightarrow{\overleftarrow{S}}$ & 弚 \\
\hline \multicolumn{2}{|c|}{ 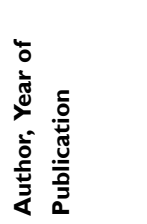 } & 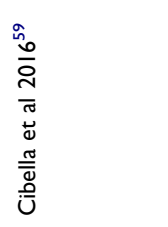 & 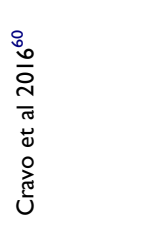 & 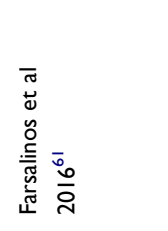 & 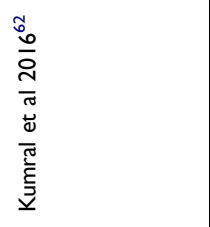 & 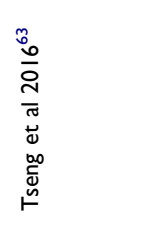 & 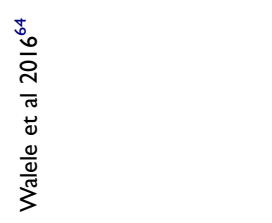 \\
\hline
\end{tabular}




\begin{tabular}{|c|c|c|c|c|c|}
\hline 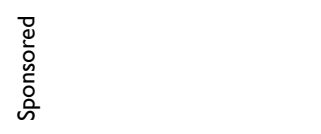 & 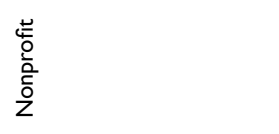 & 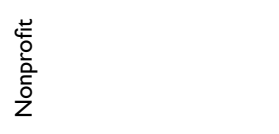 & 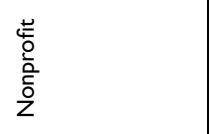 & 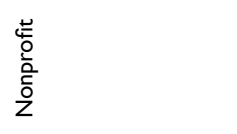 & 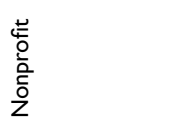 \\
\hline 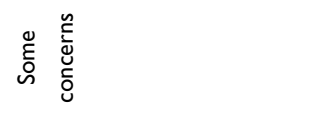 & 号 & 总 & 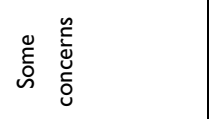 & 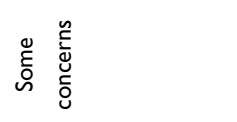 & 亳 \\
\hline $\begin{array}{l}\stackrel{\check{\Phi}}{\Xi} \\
\underline{3}\end{array}$ & 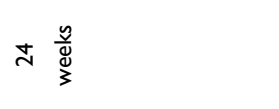 & 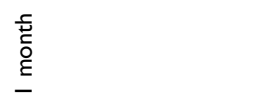 & 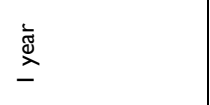 & 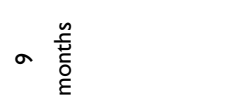 & ○ \\
\hline 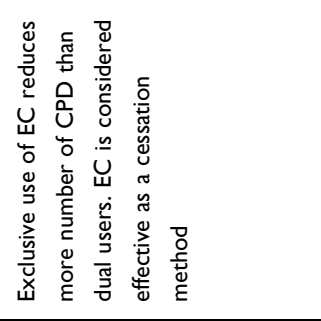 & 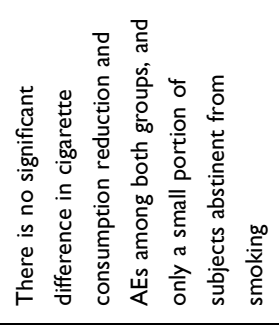 & 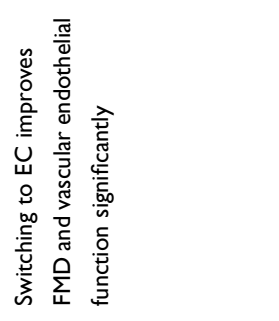 & 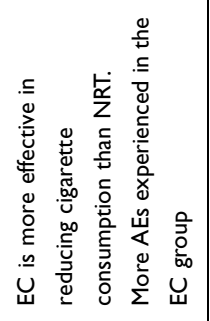 & 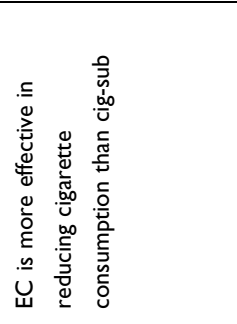 & 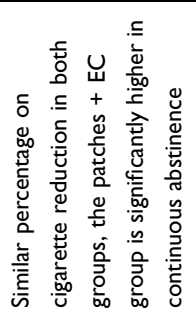 \\
\hline$>$ & $>$ & $>$ & $>$ & 1 & $>$ \\
\hline 1 & $>$ & 1 & $>$ & 1 & $>$ \\
\hline 1 & $>$ & 1 & $>$ & $>$ & $>$ \\
\hline 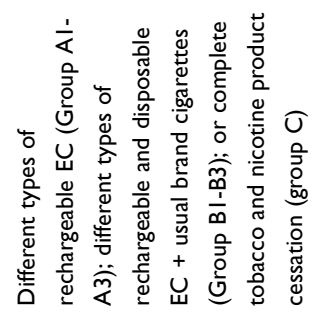 & 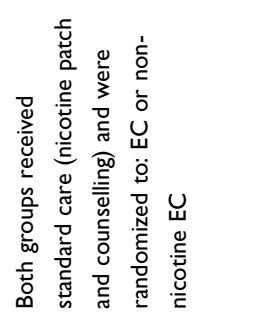 & 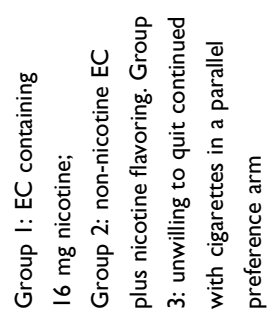 & 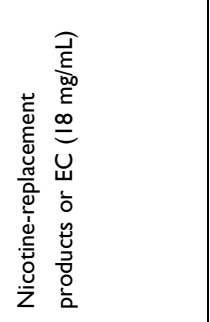 & 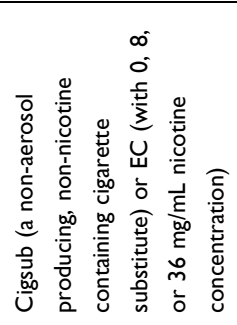 & 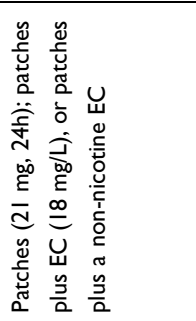 \\
\hline$\underline{\underline{n}}$ & q & $\stackrel{\Xi}{=}$ & œ & ๙ิ & $\stackrel{ \pm}{\Xi}$ \\
\hline 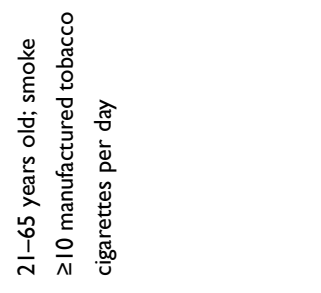 & 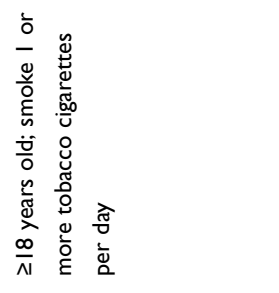 & 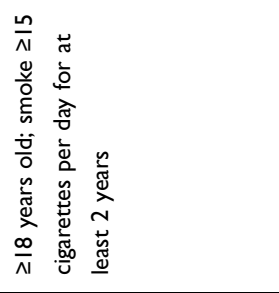 & 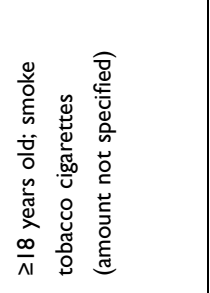 & 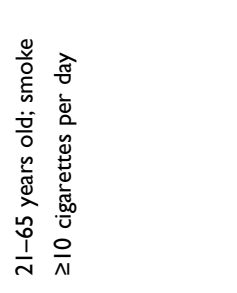 & 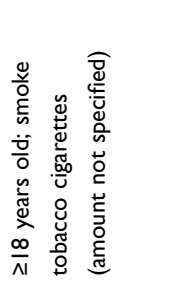 \\
\hline 芯 & 芯 & 弚 & 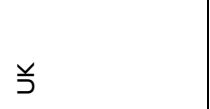 & گ્ડ & Z \\
\hline 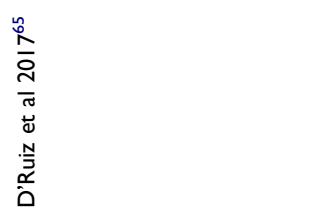 & 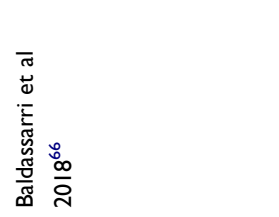 & 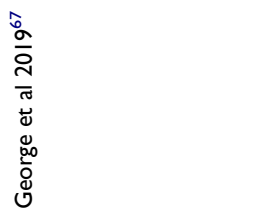 & 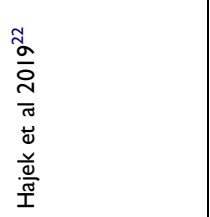 & 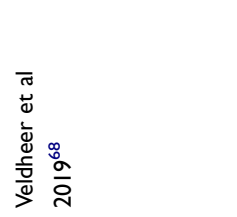 & 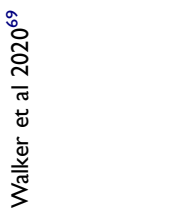 \\
\hline
\end{tabular}




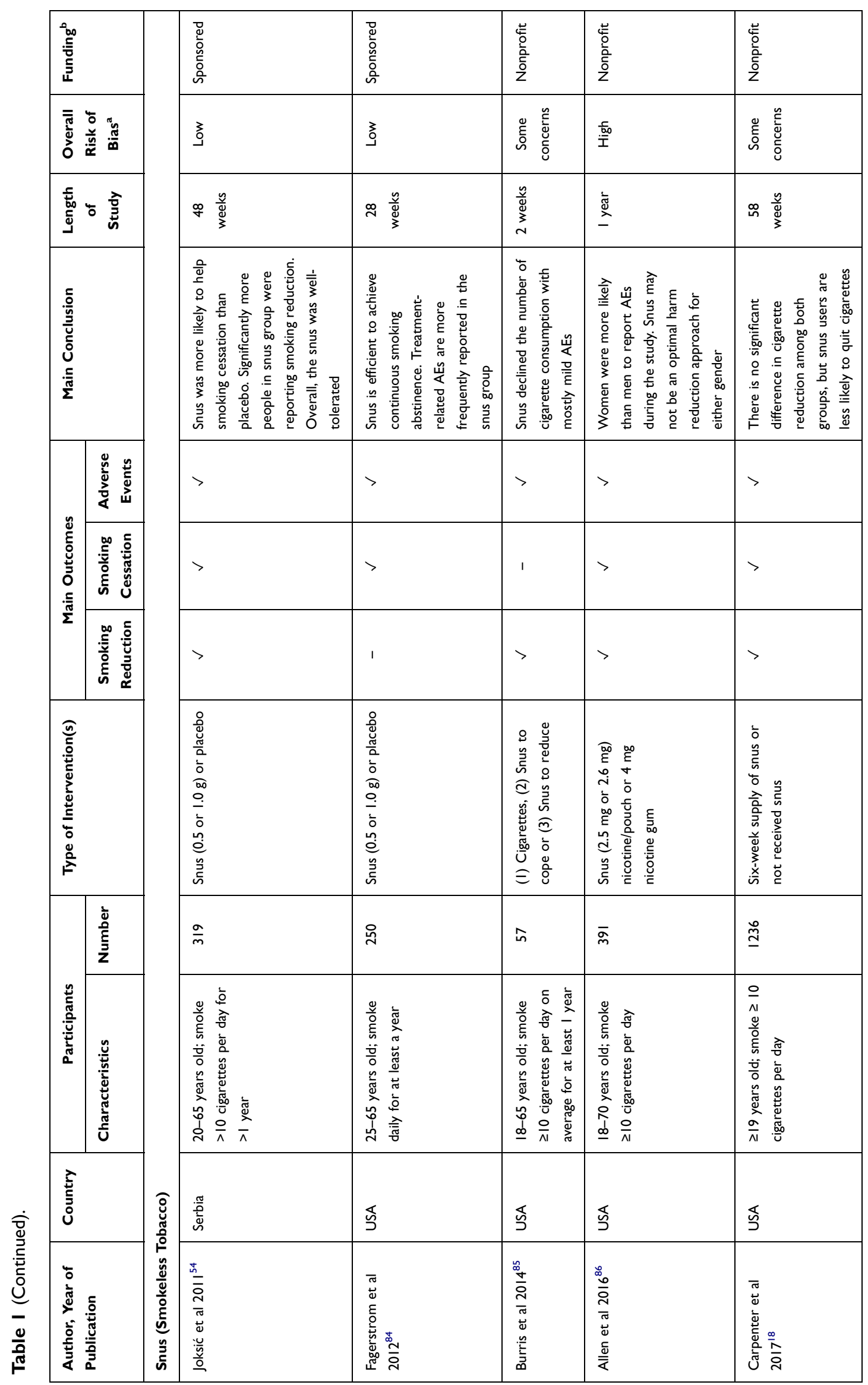




\begin{tabular}{|c|c|c|c|c|c|c|}
\hline & 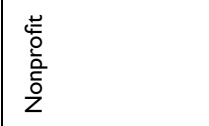 & 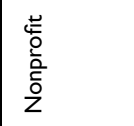 & $\overleftarrow{z}$ & 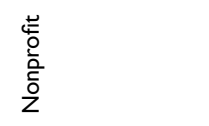 & 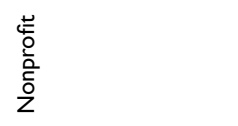 & 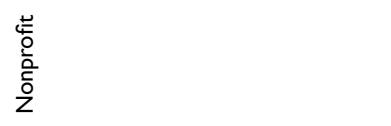 \\
\hline & 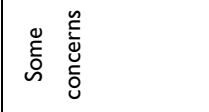 & 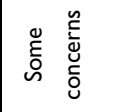 & 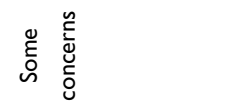 & 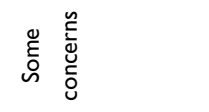 & 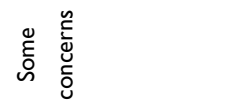 & 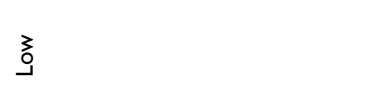 \\
\hline & 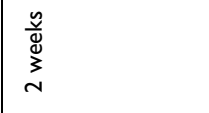 & 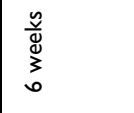 & 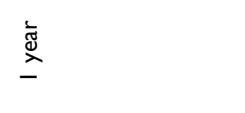 & 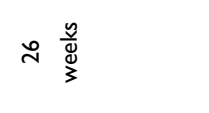 & 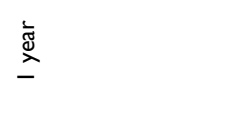 & ○ \\
\hline & 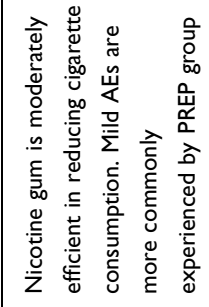 & 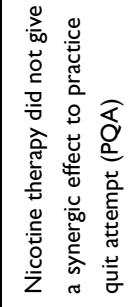 & 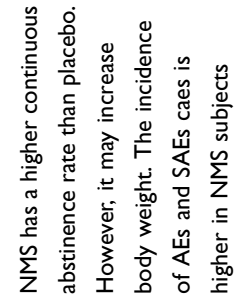 & 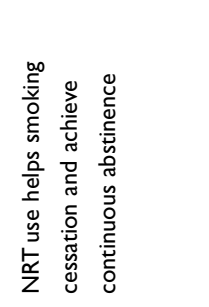 & 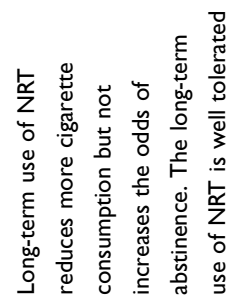 & 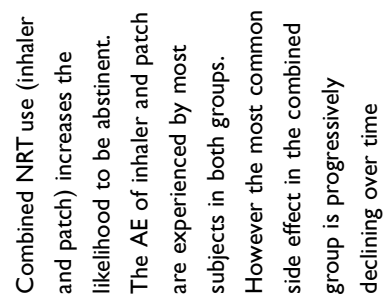 \\
\hline & $>$ & 1 & $>$ & 1 & $>$ & $>$ \\
\hline & 1 & $>$ & $>$ & $>$ & $>$ & $>$ \\
\hline & $>$ & 1 & 1 & 1 & $>$ & 1 \\
\hline & 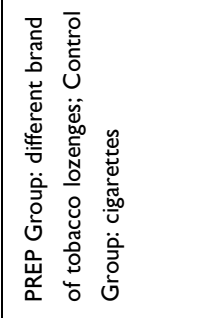 & 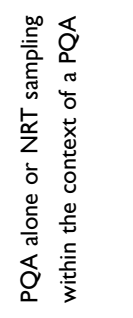 & 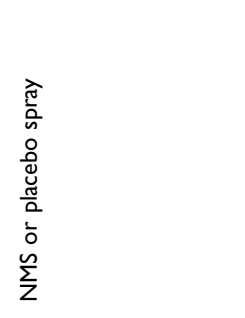 & 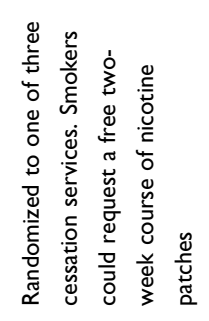 & 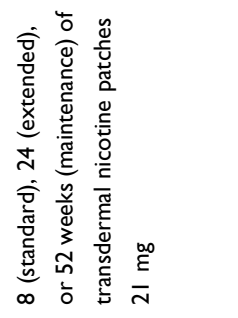 & 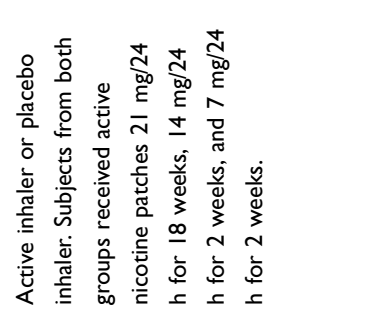 \\
\hline & $\bar{m}$ & $\underset{\infty}{\stackrel{Q}{O}}$ & $\stackrel{q}{q}$ & 志 & $\stackrel{\sim \sim \sim}{\sim}$ & 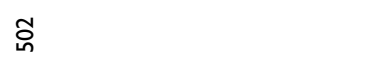 \\
\hline $\begin{array}{l}\text { E } \\
\frac{\alpha}{z}\end{array}$ & 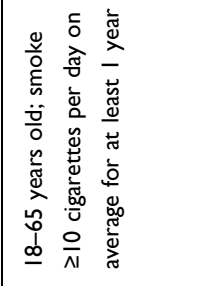 & 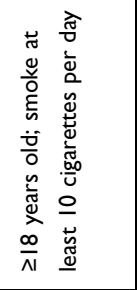 & 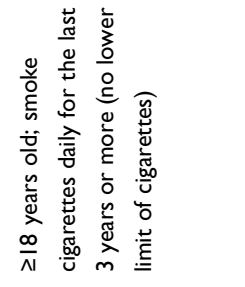 & 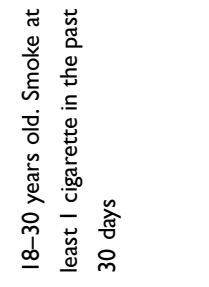 & 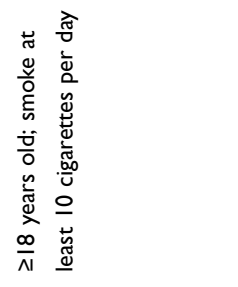 & 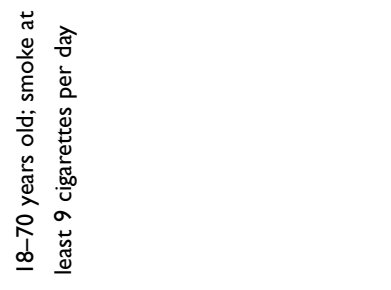 \\
\hline 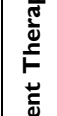 & $\overleftrightarrow{\overleftarrow{S}}$ & 吕 & 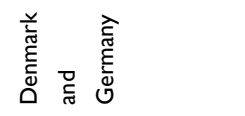 & 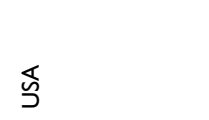 & 芯 & 竞 \\
\hline 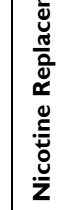 & 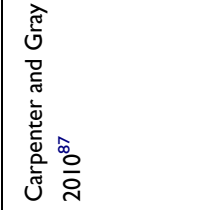 & 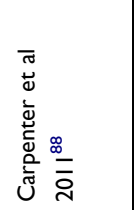 & 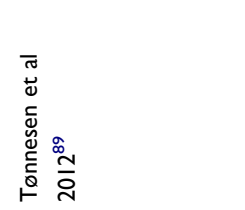 & 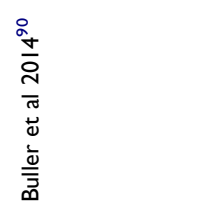 & 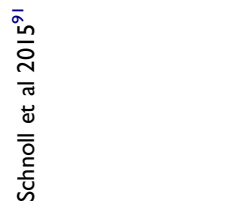 & 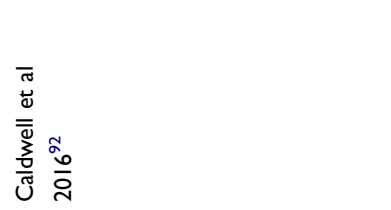 \\
\hline
\end{tabular}




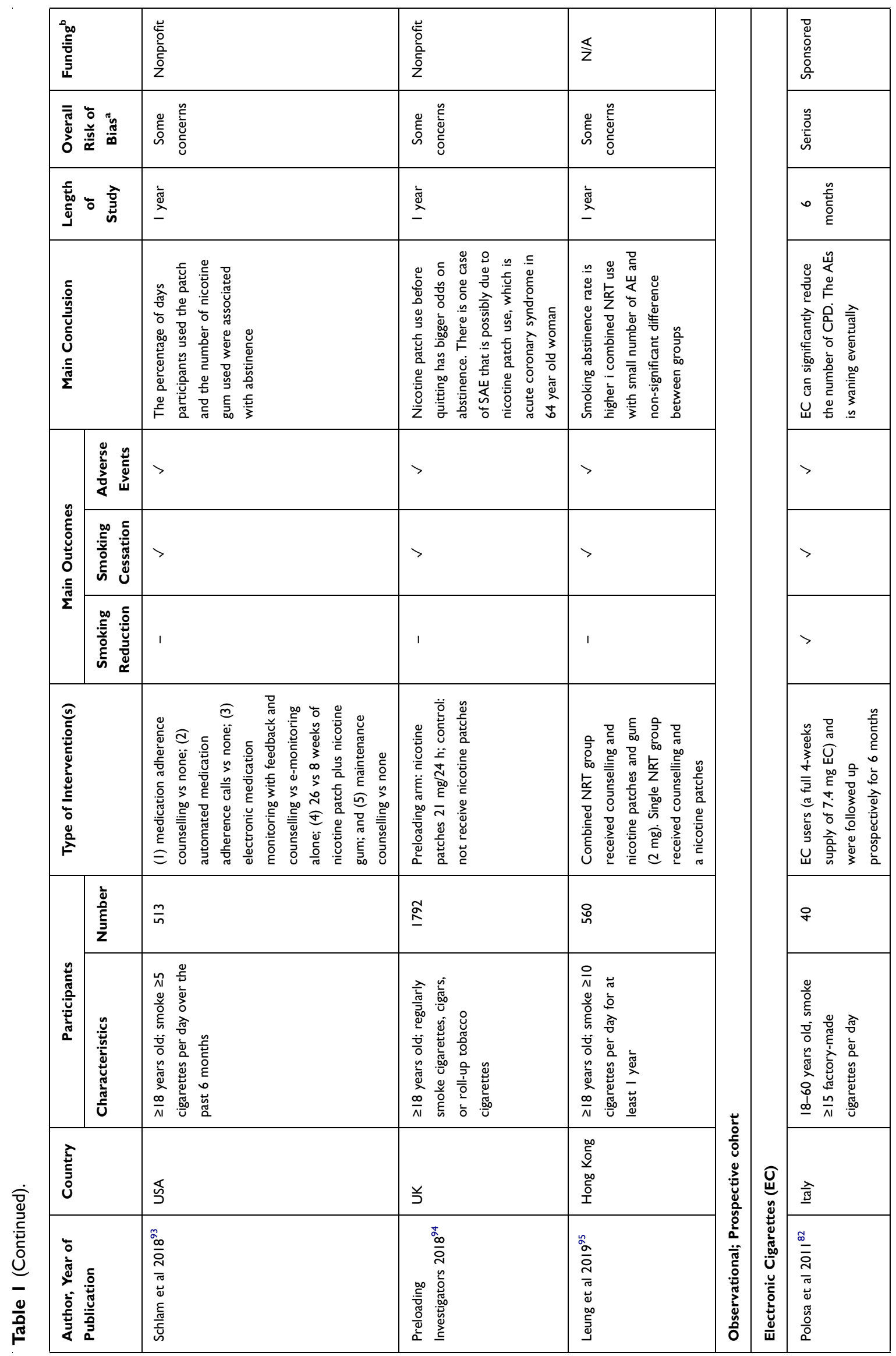




\begin{tabular}{|c|c|c|c|c|c|c|c|}
\hline 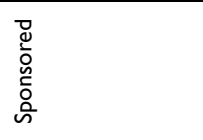 & $\begin{array}{l}\overline{0} \\
\overline{0} \\
\overline{0} \\
\overline{0} \\
0 \\
0\end{array}$ & 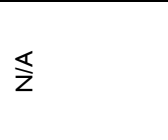 & $\overleftarrow{z}$ & 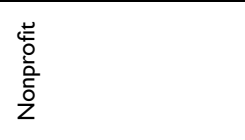 & 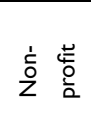 & 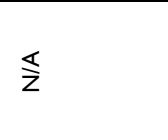 & 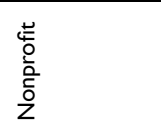 \\
\hline $\begin{array}{l}\frac{n}{o} \\
\stackrel{0}{\bar{D}} \\
\stackrel{n}{n}\end{array}$ & $\begin{array}{l}\stackrel{n}{o} \\
\frac{0}{\bar{D}} \\
\infty\end{array}$ & 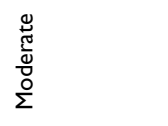 & $\begin{array}{l}\frac{n}{0} \\
\stackrel{0}{\bar{D}} \\
\infty\end{array}$ & 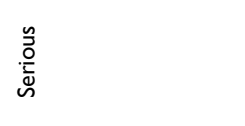 & 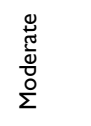 & 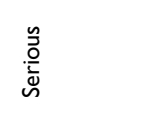 & 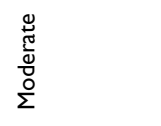 \\
\hline 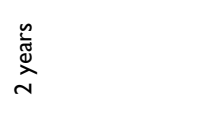 & ○ & 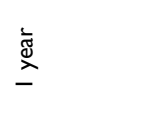 & 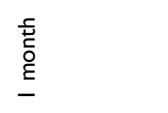 & 离 & $\stackrel{\text { ङू }}{\stackrel{\bar{\Xi}}{-}}$ & م. & 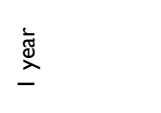 \\
\hline 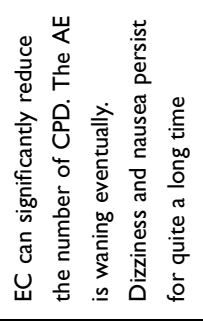 & 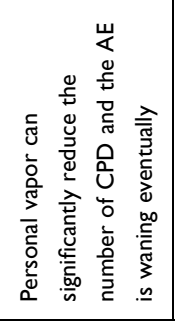 & 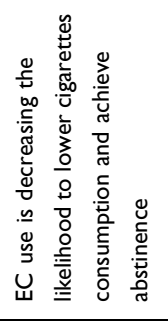 & 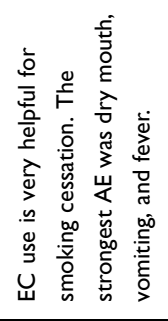 & 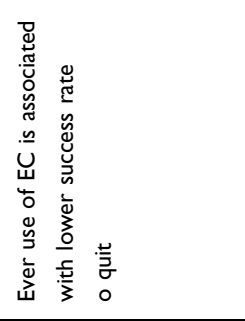 & 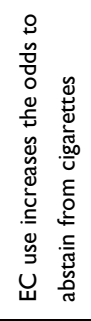 & 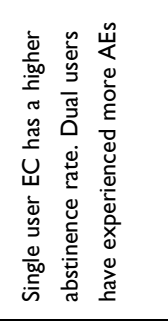 & 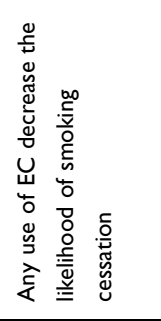 \\
\hline$>$ & $>$ & 1 & $>$ & 1 & 1 & $>$ & 1 \\
\hline$>$ & $>$ & $>$ & $>$ & $>$ & $>$ & $>$ & $>$ \\
\hline$>$ & $>$ & $>$ & 1 & $>$ & 1 & 1 & 1 \\
\hline 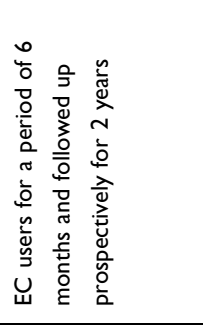 & 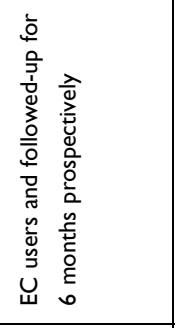 & 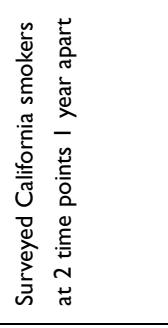 & 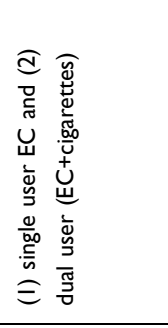 & 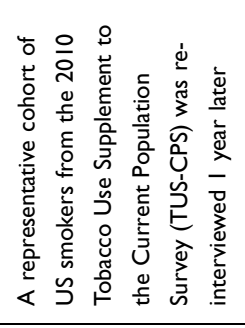 & 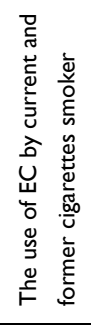 & 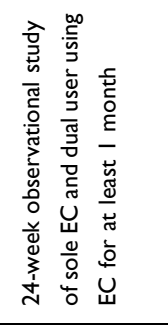 & 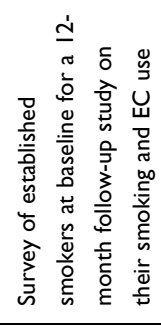 \\
\hline q & 요 & ঃ & తి & 峖 & $\hat{~}$ & $\frac{\infty}{N}$ & $\stackrel{ \pm ્ \Phi}{\simeq}$ \\
\hline 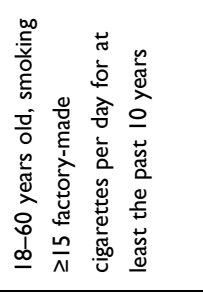 & 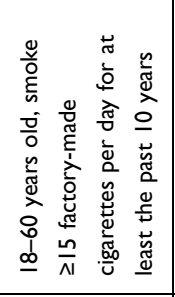 & 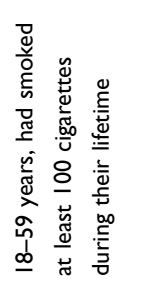 & 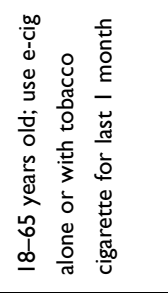 & 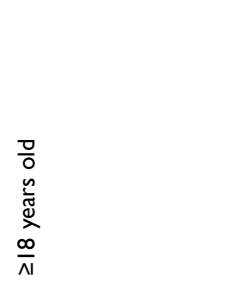 & 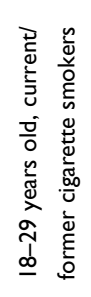 & 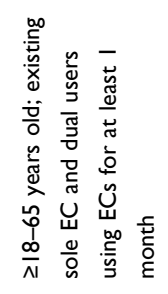 & 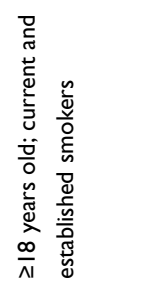 \\
\hline 롶 & ब्र & 崩 & $\begin{array}{l}\frac{\pi}{\sqrt{n}} \\
\frac{\widehat{I}}{\Sigma} \\
\frac{\pi}{\Sigma}\end{array}$ & ک & 芯 & 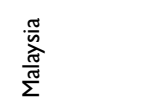 & 刹 \\
\hline 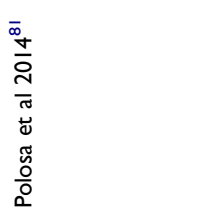 & 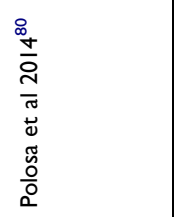 & 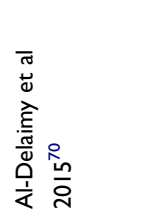 & 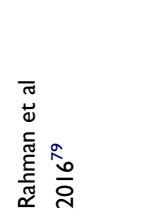 & 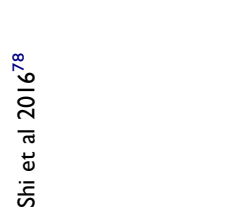 & 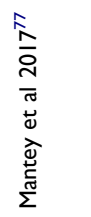 & 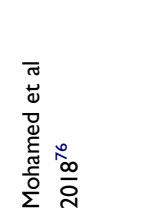 & 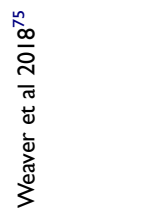 \\
\hline
\end{tabular}




\begin{tabular}{|c|c|c|c|c|c|c|}
\hline \multicolumn{2}{|c|}{ 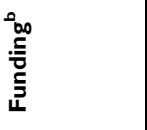 } & 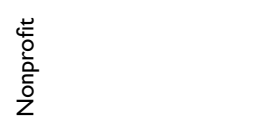 & 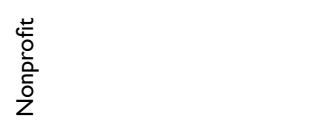 & 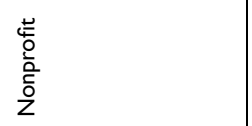 & 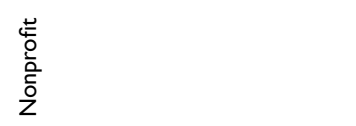 & 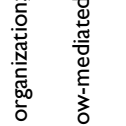 \\
\hline \multicolumn{2}{|c|}{ 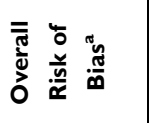 } & $\begin{array}{l}\stackrel{n}{0} \\
\frac{0}{\bar{\nu}} \\
\stackrel{\omega}{n}\end{array}$ & 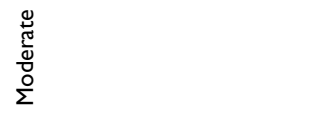 & 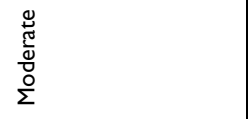 & 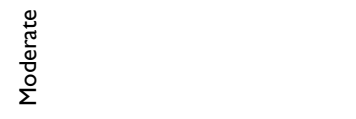 & 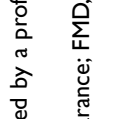 \\
\hline \multicolumn{2}{|c|}{ 䓌 } & 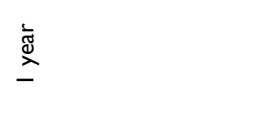 & 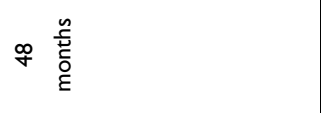 & 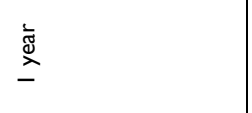 & 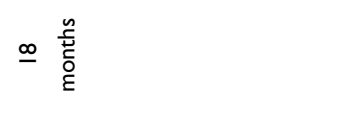 & 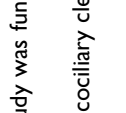 \\
\hline \multicolumn{2}{|c|}{ 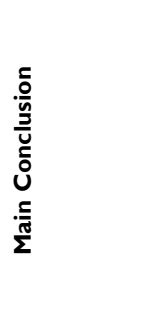 } & 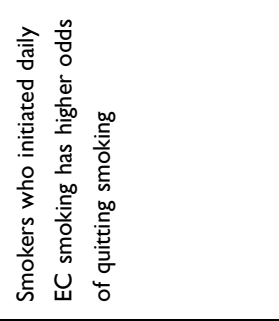 & 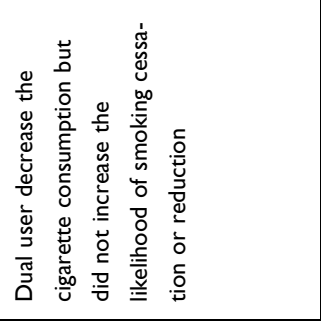 & 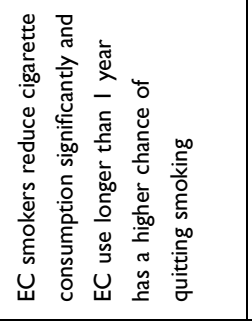 & 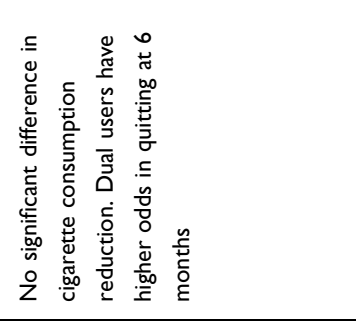 & 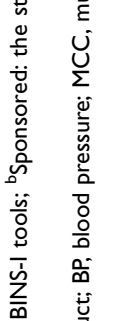 \\
\hline \multirow{3}{*}{ 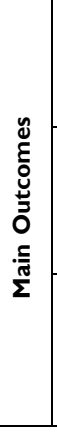 } & 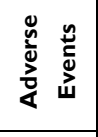 & 1 & 1 & 1 & 1 & 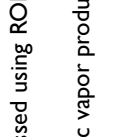 \\
\hline & 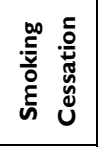 & $>$ & $>$ & $>$ & $>$ & 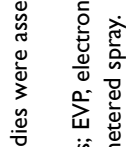 \\
\hline & 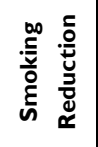 & $>$ & $>$ & $>$ & $\stackrel{m}{i}$ & 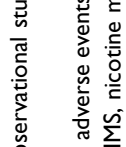 \\
\hline \multicolumn{2}{|c|}{ 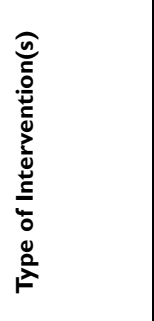 } & 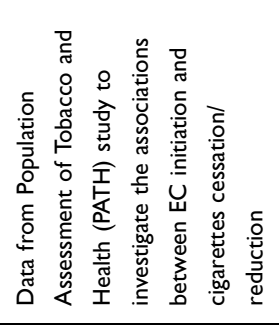 & 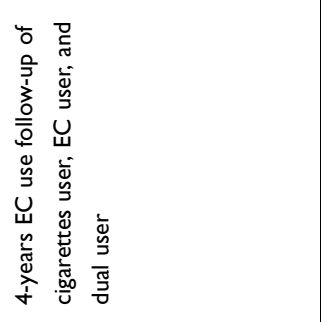 & 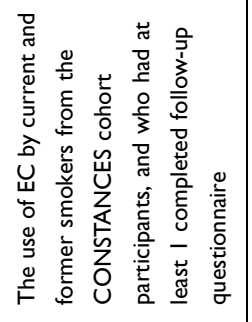 & 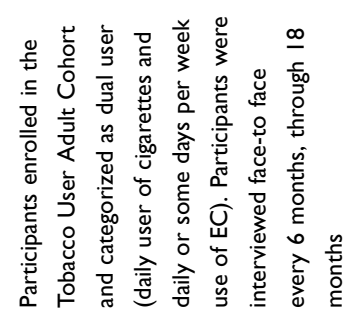 & 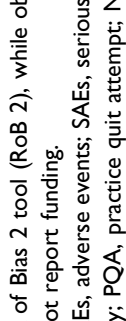 \\
\hline \multirow[b]{2}{*}{ 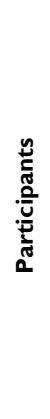 } & $\begin{array}{l}\text { ऐ̀े } \\
\text { है } \\
\bar{z}\end{array}$ & $\stackrel{ \pm}{\frac{ \pm}{n}}$ & $\frac{n}{\alpha}$ & $\stackrel{\stackrel{\sim}{\sim}}{N}$ & $\frac{1}{6}$ & 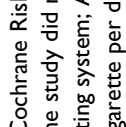 \\
\hline & 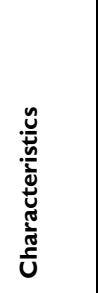 & 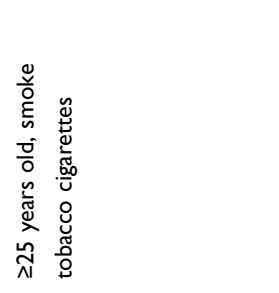 & 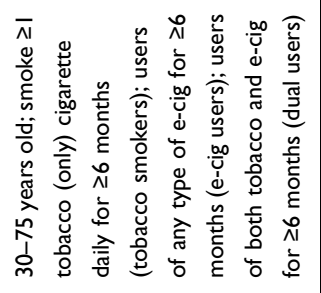 & 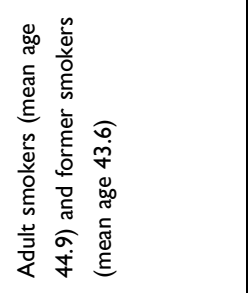 & 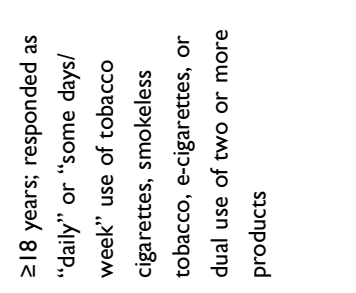 & 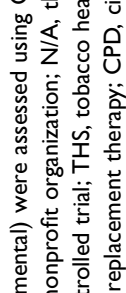 \\
\hline \multicolumn{2}{|l|}{ 害 } & 凹్ & 츨 & 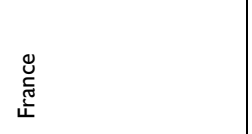 & 杀 & 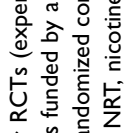 \\
\hline \multicolumn{2}{|c|}{ 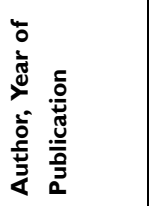 } & 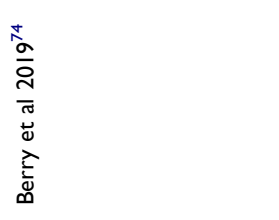 & 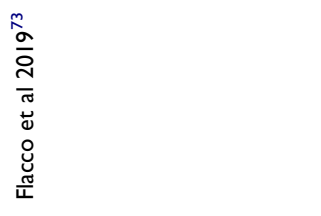 & 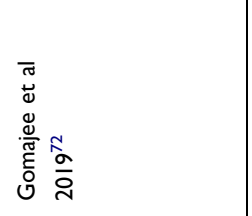 & 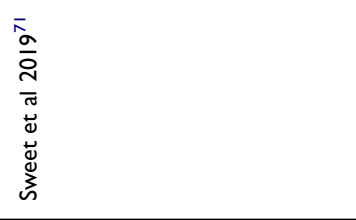 & 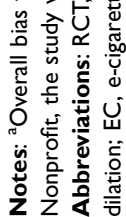 \\
\hline
\end{tabular}


with a higher likelihood of smoking abstinence and that EC increased the odds to abstain from cigarettes, ${ }^{71-74,76,77,79-82}$ while three cohort studies suggested that any EC users may be at increased risk for smoking cessation failure and that the use of EC was associated with lower success rate to quit smoking, ${ }^{70,75,78}$ with reported adjusted odds ratio (aOR) $0.41 ; 95 \%$ confidence interval (CI): $0.186-0.93,{ }^{70}$ aOR 0.4 , 95\% Cl: $0.2-0.8^{78}$ and aOR $0.25,95 \% \mathrm{CI}(0.11-0.57){ }^{75} \mathrm{In}$ addition, three studies showed that single user EC had much higher odds to abstain from cigarette consumption than dual users (EC+ cigarette) ${ }^{73,76,79}$ In addition, there was variation in the length of observed abstinence among the included prospective cohort studies, ranging from seven days to 12 months follow-up.

Studies on snus as a smoking cessation method were also unequivocal in their conclusion of whether snus can be an efficient harm reduction approach. Two studies showed that snus was approximately two to three times more efficient in attaining continuous abstinence compared to placebo $^{54,84}$ while two other studies suggested that snus could reduce the likelihood to quit smoking and that snus may not be an ideal way in reducing tobacco harm. ${ }^{18,86}$ Moreover, overall NRT use showed that they helped in sustaining smoking abstinence and that two studies ${ }^{92,95}$ observed a synergetic effect of NRT combination in abstinence rate. More details on study outcomes, including length of abstinence in each study, are provided in Table S1.

\section{Adverse Events}

Adverse events were either self-reported or laboratorymeasured. Almost all RCTs studies in the EC group were assessing its potential adverse events (14 out of 16 studies). ${ }^{22,55,56,58-67,69}$ In addition, five out of 13 prospective cohort studies on EC also reported adverse events. $^{76,79-82}$ The most frequently reported adverse events in the EC group were classified as mild, such as cough, mouth, and throat irritation, headache, difficulty sleeping, and abnormal dreams. Two studies reported the incidence rate of adverse events of nicotine EC compared to nicotine patches $(1.05,95 \% \mathrm{CI}: 0.82-1.34)^{55}$ and incidence rate associated with the use of EC $(1.60,95 \% \mathrm{CI}: 1.55$ -1.65). ${ }^{60}$ Overall results indicated no significant intervention-related severe adverse events. Similar results were also observed in EC cohort studies, that the most frequently reported adverse events were moderate eg, mouth and throat-related problems. The only included HNB study reported the safety profile of tobacco heating system (THS) and indicated only moderate adverse events as well, for instance, headache, oropharyngeal pain, and abnormal spirometry, with the estimated incidence of $62.5 \%$ in THS and $70.7 \%$ in the cigarette group. ${ }^{83}$ Moreover, all studies assessing snus reported interventionrelated adverse events. Four studies comparing smokers who were randomized to use snus and no snus (identified as control or placebo) showed that the adverse events were more frequently reported in the snus group compared to the control group. ${ }^{18,54,84,85}$ The adverse events were mostly considered mild eg, nausea, burning in throat, and mouth, and stomach problems. One study assessed the use of snus vs nicotine gum for different gender showed that women were more likely to inform adverse events during the study than men. ${ }^{86}$

In addition, three NRT studies that assessed the use of tobacco lozenges ${ }^{87}$ nicotine metered spray, ${ }^{89}$ and nicotine patches $^{94}$ described that the adverse events were more common in the intervention group rather than in the control or placebo group. The reported adverse events were mostly mild, however one study suggested the occurrence of severe adverse events ie, acute coronary syndrome, which was possibly related to the use of nicotine patches. ${ }^{94}$ The remaining studies on NRT suggested rather moderate adverse events. ${ }^{91-93,95}$

\section{Risk of Bias and Quality Assessment}

Across the 44 articles included in this review, 31 studies were RCTs. According to study design classification in the RoB 2 tool, 29 studies ${ }^{18,22,54-57,59-63,65-69,83-95}$ were classified as parallel-group trials and the remaining two were categorized as crossover trials. ${ }^{58,64}$ Based on RoB tool assessment, 22 studies out of 31 included RCTs (71\%) had some risk of bias, two studies had high risk of bias $(6 \%)$ and seven studies were deemed low in their risk of bias assessment (23\%). In details, $96 \%$ of studies had low risk through a selection of the reported result, $96 \%$ studies had low risk from measurement of the outcome, $78 \%$ had low risk of bias from missing outcome data, $70 \%$ were assessed as had a low risk of bias through deviations from intended interventions, and $44 \%$ studies had low risk of bias from randomization process. Figure 2 depicts the risk of bias assessment in these 30 included RCTs.

According to ROBINS-I tool to assess the risk of bias in the included observational studies, seven out of 13 studies (54\%) had serious risk of bias and six studies had moderate risk of bias $(46 \%)$. The overall risk of bias assessment from RoB 2 and ROBINS-I can be seen in Table 1. 


\section{Discussion}

This systematic review described the utilization of alternative tobacco and nicotine products in terms of assisting current cigarette smokers in reducing their daily cigarette consumption, and smoking cessation by tempering withdrawal symptoms. This review also defined the potential adverse events that could occur due to using different types of alternative tobacco and nicotine products. Overall, the results indicated that the use of alternative tobacco and nicotine products had the potential to encourage smoking reduction by decreasing the number of cigarettes the current smokers used, even though variations in the efficacy of different products were observed. EC with or without nicotine, snus, and NRT were observed to have a moderate effect in the smoking reduction. ${ }^{18,22,55-}$ 57,59,63,66,68-74,78,80-82,85-87,91 Moreover, the effectiveness of alternative tobacco and nicotine products on smoking cessation was consistently observed in almost all included experimental and observational studies, even though the abstinence verification method and the degree of effectiveness were varied among different products. Even if the effectiveness is considered moderate, the use of these products was observed to associate with a reduction in the number of cigarettes used, which is prominent in highlighting the substantial evidence demonstrating that gradual reduction in cigarette consumption could further initiate future quit attempts. ${ }^{38}$
Among all alternative tobacco and nicotine products, EC was the most frequently examined product to aid reduction/cessation among the included studies $(n=18 /$ 44). In addition to helping relieve nicotine withdrawal similar to other interventions, EC use was regarded as an effective behavioral substitute, as it addressed additional sensory and behavioral cues of smoking. ${ }^{35,55}$ The existing literature suggested that EC might be helpful in reduction/ cessation attempts, with several RCTs consistently reporting EC as superior compared to the NRT. ${ }^{22,55}$ Another recent trial conducted in the context of the stop smoking service in England further demonstrated that using EC as a cessation aid was likely to be cost-effective compared to NRT in the same setting. It generated a significantly higher one-year quit rate and incurred lower costs than NRT. ${ }^{96}$ Further studies comparing clinical efficacy, as well as the economic benefits of these interventions, are required to determine the generalizability of this finding.

Similar to previous reviews, we found that short to medium-term use of EC was associated with few adverse events, of which the large majority were considered nonserious. ${ }^{17,38,58,60,63-66,76}$ The longest follow-up period was observed in a study by Polosa et al, showing that EC was well-tolerated during six-months use with no major adverse event (requiring hospitalization/unscheduled primary care consultation) occurring during the two-year 
follow-up period. ${ }^{81}$ In addition, potential pulmonary and cardiovascular benefit was observed in a study where cigarette smokers changed partly or completely to EC for five days, ${ }^{65}$ suggesting potential for harm reduction. Nevertheless, clinical evidence on long-term impact has yet to be characterized. Several studies have shown that toxicants generated from filler (eg, glycerol, polyglycerol) and nicotine inhalation in ECs might contain carcinogens, oxidants, and irritants, such as formaldehyde, acetaldehyde, methylglyoxal, and acrolein, ${ }^{97,98}$ and its chronic exposure has been associated with inflammation. ${ }^{99}$ Yet, previous study showed that compared with combustible tobacco products, ECs contain a significantly lower level of toxicants. ${ }^{100}$ Understanding whether lower exposure to these toxicants will result in tangible long-term health benefit/harm is urgently required.

Although the findings from this review indicated that ECs might be one of the potential strategies in tobacco harm reduction, it should be emphasized that since EC products were very diverse in both design and characteristics, the effectiveness and safety might differ as well. Exposure to nicotine and other potentially toxic substances in ECs was varied and depended largely on product characteristics, such as liquid constituents, device characteristics, and settings. ${ }^{101,102}$ In addition, the risk of smoking relapse in a former smoker using ECs remains unclear. The most recent systematic review and meta-analysis study showed an increased risk of smoking relapse among EC users (RR 1.38 (95\%CI: 1.11-1.65)). However, this pooled estimate was based on very few studies (three studies in the quantitative analysis). ${ }^{103}$ Therefore, more studies are needed to further confirm this finding.

Besides, the included studies primarily focused on the potential effectiveness of ECs for smoking reduction and cessation in adult smokers, when in reality, these products were also being used by youth, possibly those who had never tried cigarettes. ${ }^{102}$ There are also increasing public health concerns that ECs may renormalize "smoking-like" behavior, particularly among youth. The prevalence of EC use among adolescent populations is currently increasing, posing a concern whether ECs are exposing this vulnerable group to nicotine. Although there is evidence that ECs are considered safer in comparison to cigarettes, the early exposure to nicotine may predict concerning patterns of future nicotine use. In addition, there have been reports of pulmonary risks such as the condition electronic-cigarette-associated lung injury (EVALI) and neurodevelopmental effects. ${ }^{104}$ Furthermore, very little evidence existed on EC as a smoking cessation approach among youth. $^{105}$

In order to assess EC as one of the tobacco harm reduction approaches at a population level, a thorough understanding of the estimation of both potential benefits and harms from EC should also be taken into account. In addition, the huge variation in terms of length of studies and the number of participants in the included studies in this review suggested that more well-designed RCTs and observational studies are needed to further clarify our findings.

Studies on the use of snus for smoking reduction were not unequivocal in their conclusion whether snus can be an efficient smoking reduction/cessation approach. ${ }^{18,85,86}$ In one of the largest trials involving 1236 participants that reported snus decreased quit attempts, the provision of snus was unguided without any additional support. ${ }^{18}$ The importance of behavioral support as an adjunct for smoking reduction/cessation programs has been underlined by a previous Cochrane review by Hartmann-Boyce et al. ${ }^{106}$ This review showed high-certainty evidence that provision of behavioral support (eg, group therapy/individual counseling, either in-person or by telephone; written material), in addition to pharmacotherapy was likely to increase quit attempts by up to $20 \%$, based on pooled estimates from 65 trials. $^{106,107}$

Previous meta analysis comprising two RCTs investigating effectiveness of snus showed that snus increased quit rates. ${ }^{108}$ Nevertheless, both trials had relatively small sample sizes ( $\mathrm{n}=250$ and 319$)$, and both success rates were relatively low. ${ }^{54,64}$ Snus had been reported as the preferred method for quitting in Scandinavian countries, ${ }^{109-111}$ and previous observational studies using self-reported data confirmed that compared with the NRT, snus increased probability of cessation, ${ }^{112}$ presumably owing to the nicotine uptake from snus that resembles that of combustible tobacco compared to latter approach. ${ }^{112-114}$ Nevertheless, the degree of evidence was low, suggesting further research is currently needed to expand the evidence base for the utility, as well as safety, of snus as a reduction/ cessation aid.

Different forms and delivery methods of NRT had been evaluated by the included studies, including patches, ${ }^{90,92,95}$ inhaler, ${ }^{92}$ mouth sprays, ${ }^{89}$ gum/lozenges, ${ }^{95}$ or comparison of different forms. ${ }^{92,95}$ Combined NRT products had been consistently reported as superior to assist cessation up to 52 weeks ${ }^{95,115-117}$ and that NRT can increase the chance to successfully stop smoking. ${ }^{17}$ A previous review showed 
that combination NRT produced greatest benefits relative to monotherapy for smoking cessation. ${ }^{118}$ It has been estimated that the use of different forms of NRT resulted in more adequate nicotine replacement from various mechanisms, possibly generating additive effects (ie, patch releases steady-state nicotine serum level to prevent acute withdrawal, while another NRT form, eg, gum/ lozenge, may provide a coping mechanism addressing the behavioral urge of smoking). ${ }^{49,116,117}$ Our restriction to include references in the last 10 years might be limiting the effectiveness of NRT in smoking reduction/cessation because the availability of NRT products and their role had been assessed extensively since as early as the $1980 \mathrm{~s} .{ }^{17}$ However, the reason for our restriction was to showcase and focus on the most recent evidence on alternative tobacco and nicotine products.

As nicotine may interfere with the cardiovascular system, presumably through sympathetic neural stimulation and systemic catecholamine release, cardiovascular safety profile associated with NRT use has been extensively examined. $^{23,89,91,93-95,119-121}$ Relatively long-term (fiveyear observation period) impact of NRT gum use was assessed by Murray et al, which showed that long-term exposure of this intervention was not associated with major adverse cardiovascular events. ${ }^{119}$ Among patients with a history of acute coronary syndrome, no excess risk of recurrent cardiovascular events was observed following the use of NRT in this high-risk population. ${ }^{121}$ As NRT delivers nicotine without a combustion process, the risk might be lower compared to that of EC, ${ }^{23}$ nevertheless, current evidence remains unclear with regard to this comparison, and further robust investigation is required.

Alternative tobacco and nicotine products, including EC, smokeless tobacco, and NRT, are a current development in tobacco harm reduction. ${ }^{23,122}$ According to evidence gathered by this review as well as previous research, ${ }^{17,23,34,36,38,40}$ these approaches can be considered to be less harmful in a means of causing fewer adverse events compared to a cigarette and has the potential to assist smoking reduction and even cessation. Among smokers, the urge to smoke is often tough to break, and relapse is prevalent even for those who intend to quit smoking. ${ }^{56}$ Therefore, to prevent tobacco-related morbidity and mortality, there is an urgency for alternative and more efficient means to reduce the harms caused by a particular behaviour. The approach in tobacco harm reduction includes amending and adjusting regulations that potentially can escalate damages, empowering people and policymakers with accurate information and evidence-based policy, and suggesting alternatives and substitutions of lower-risk products that may further promote the cessation of cigarette smoking to current smokers. ${ }^{123}$ In addition, to ensure efficient resource allocation for such policy, more economic evaluation studies comparing clinical effectiveness and cost of different alternative tobacco and nicotine products would be valuable to support evidence-based public health initiatives. ${ }^{96}$

Furthermore, the strength of our review lies in the provision of comprehensive information on the role of different types of alternative tobacco and nicotine products in smoking reduction/cessation, as well as their potential safety issues. In addition, we used extensive search strategies, resulting in a relatively large number of included studies. Nevertheless, this review also has limitations. First, due to heterogeneity of the included studies, we were unable to conduct a metaanalysis, nevertheless a narrative review has been provided outlining current evidence on this topic and highlighting gap that remains unexplored for future studies. Another limitation was the risk of publication bias since we did not search grey literature, as we only included peer-reviewed published studies to ensure comparable study quality.

\section{Conclusion}

The results suggest that the use of alternative tobacco and nicotine products has been shown to potentially influence smoking reduction and cessation process, with various degree of effectiveness between different products. Available evidence indicated that these products are generally well-tolerated following short to medium-term use. The most common adverse events reported included mouth and throat-related irritation, dry cough, headache, and changes in pulmonary laboratory functions which were considerably milder than with conventional cigarettes. These findings are also highlighting the potential role of these products in a tobacco harm reduction approach. Further studies should focus on investigating long-term outcome, safety and effectiveness of alternative tobacco and nicotine products and also on monitoring both product use and awareness to better inform smoking reduction/cessation policy.

\section{Data Sharing Statement}

All data generated or analyzed during this study are included in this published article and Table S1. 


\section{Author Contributions}

Study conception and design: NZ, FVP, WNI, AAS; search strategy: NZ, FVP, AAS; study screening: NZ, FVP, WNI, AAS; data organization and presentation: NZ, FVP, RA, MIB, IMP; appraisal of included studies: NZ, FVP, WNI; data analysis and interpretation: NZ, FVP, WNI, RA, AAS; initial manuscript drafting: NZ, WNI; manuscript review and finalization: NZ, FVP, WNI, RA, IMP, MIB, RL, AA, AAS. All authors contributed to data analysis, drafting or revising the article, have agreed on the journal to which the article will be submitted, gave final approval of the version to be published, and agree to be accountable for all aspects of the work.

\section{Funding}

This research was funded by the Center of Excellence in Higher Education for Pharmaceutical Care Innovation, Universitas Padjadjaran.

\section{Disclosure}

The authors reported no conflicts of interest in this work.

\section{References}

1. O'Keeffe LM, Taylor G, Huxley RR, Mitchell P, Woodward M, Peters SAE. Smoking as a risk factor for lung cancer in women and men: a systematic review and meta-analysis. BMJ Open. 2018;8(10): e021611. doi:10.1136/bmjopen-2018-021611

2. Hackshaw A, Morris JK, Boniface S, Tang JL, Milenković D. Low cigarette consumption and risk of coronary heart disease and stroke: meta-analysis of 141 cohort studies in 55 study reports. BMJ. 2018;360:j5855. doi:10.1136/bmj.j5855

3. Jones-Burton C, Seliger SL, Scherer RW, et al. Cigarette smoking and incident chronic kidney disease: a systematic review. Am J Nephrol. 2007;27(4):342-351. doi:10.1159/000103382

4. Terzikhan N, Verhamme KMC, Hofman A, Stricker BH, Brusselle GG, Lahousse L. Prevalence and incidence of COPD in smokers and non-smokers: the Rotterdam Study. Eur J Epidemiol. 2016;31(8):785-792. doi:10.1007/s10654-016-0132-z

5. Liu Y, Pleasants RA, Croft JB, et al. Smoking duration, respiratory symptoms, and COPD in adults aged 45 years with a smoking history. Int J COPD. 2015;10:1409-1416. doi:10.2147/COPD.S82259

6. World Health Organization. WHO report on the global tobacco epidemic 2019: offer help to quit tobacco. Geneva; 2019.

7. Jha P, Ramasundarahettige C, Landsman V, et al. 21st-century hazards of smoking and benefits of cessation in the United States. $N$ Engl $J$ Med. 2013;368(4):341-350. doi:10.1056/ NEJMsa 1211128

8. Sakata R, McGale P, Grant EJ, Ozasa K, Peto R, Darby SC. Impact of smoking on mortality and life expectancy in Japanese smokers: a prospective cohort study. BMJ. 2012;345:e7093. doi:10.1136/bmj. e7093

9. Creamer MR, Wang TW, Babb S, et al. Tobacco product use and cessation indicators among adults: United States, 2018. Morb Mortal Wkly Rep. 2019;68:1013-1019. doi:10.15585/mmwr. $\mathrm{mm} 6845 \mathrm{a} 2$
10. Hughes JR, Keely J, Naud S. Shape of the relapse curve and long-term abstinence among untreated smokers. Addiction. 2004;99(1):29-38. doi:10.1111/j.1360-0443.2004.00540.x

11. Stead LF, Carroll AJ, Lancaster T. Group behaviour therapy programmes for smoking cessation. Cochrane Database Syst Rev. 2017;2017.

12. Stead LF, Koilpillai P, Fanshawe TR, Lancaster T. Combined pharmacotherapy and behavioural interventions for smoking cessation. Cochrane Database Syst Rev. 2016;2016.

13. Pezzuto A, Carico E. Effectiveness of smoking cessation in smokers with COPD and nocturnal oxygen desaturation: functional analysis. Clin Respir J. 2020;14(1):29-34. doi:10.1111/crj.13096

14. Pezzuto A, Stellato M, Catania G, et al. Short-term benefit of smoking cessation along with glycopirronium on lung function and respiratory symptoms in mild COPD patients: a retrospective study. $J$ Breath Res. 2018;12(4):046007. doi:10.1088/1752-7163/aad0a8

15. Etter JF. Nicotine replacement therapy for long-term smoking cessation: a meta-analysis. Tob Control. 2006;15(4):280-285. doi: $10.1136 / t c .2005 .015487$

16. Villalobos RE, Ambrocio GPL, Fernandez L. Electronic cigarettes for smoking cessation: an individual patient meta-analysis of randomized controlled trials. In: Tobacco, Smoking Control and Health Education. European Respiratory Society; 2019:OA5135.

17. Hartmann-Boyce J, Chepkin SC, Ye W, Bullen C, Lancaster T. Nicotine replacement therapy versus control for smoking cessation. Cochrane Database Syst Rev. 2018(5):CD000146.

18. Carpenter MJ, Wahlquist AE, Burris JL, et al. Snus undermines quit attempts but not abstinence: a randomised clinical trial among US smokers. Tob Control. 2017;26(2):202-209. doi:10.1136/tobaccocontrol-2015-052783

19. Tayyarah R, Long GA. Comparison of select analytes in aerosol from e-cigarettes with smoke from conventional cigarettes and with ambient air. Regul Toxicol Pharmacol. 2014;70(3):704-710. doi:10.1016/j.yrtph.2014.10.010

20. Wagner KA, Flora JW, Melvin MS, et al. An evaluation of electronic cigarette formulations and aerosols for harmful and potentially harmful constituents (HPHCs) typically derived from combustion. Regul Toxicol Pharmacol. 2018;95:153-160. doi:10.1016/j. yrtph.2018.03.012

21. Bullen C, McRobbie H, Thornley S, Glover M, Lin R, Laugesen M. Effect of an electronic nicotine delivery device (e cigarette) on desire to smoke and withdrawal, user preferences and nicotine delivery: randomised cross-over trial. Tob Control. 2010;19(2):98-103. doi:10.1136/tc.2009.031567

22. Hajek P, Phillips-Waller A, Przulj D, et al. A randomized trial of E-cigarettes versus nicotine-replacement therapy. $N$ Engl $J$ Med. 2019;380(7):629-637. doi:10.1056/NEJMoa1808779

23. Farsalinos KE, Polosa R. Safety evaluation and risk assessment of electronic cigarettes as tobacco cigarette substitutes: a systematic review. Ther Adv Drug Saf. 2014;5(2):67-86. doi:10.1177/ 2042098614524430

24. Vakkalanka JP, Hardison LS, Holstege CP. Epidemiological trends in electronic cigarette exposures reported to U.S. Poison Centers. Clin Toxicol. 2014;52(5):542-548. doi:10.3109/15563650.2014.913176

25. Vardavas CI, Anagnostopoulos N, Kougias M, Evangelopoulou V, Connolly GN, Behrakis PK. Short-term pulmonary effects of using an electronic cigarette: impact on respiratory flow resistance, impedance, and exhaled nitric oxide. Chest. 2012;141 (6):1400-1406. doi:10.1378/chest.11-2443

26. McNeill A, Brose LS, Calder R, et al. Evidence review of e-cigarettes and heated tobacco products 2018. A report commissioned by public health England. 2018.

27. Jackson SE, Hill E, Shahab L, Beard E, Michie S, Brown J. Prevalence and correlates of long-term e-cigarette and nicotine replacement therapy use: a prospective study in England. $B M J$ Open. 2019;9(10):e029252. doi:10.1136/bmjopen-2019-029252 
28. Mirbolouk M, Charkhchi P, Kianoush S, et al. Prevalence and distribution of E-cigarette use among U.S. adults: behavioral risk factor surveillance system, 2016. Ann Intern Med. 2018;169 (7):429. doi:10.7326/M17-3440

29. Brown J, West R, Beard E, Michie S, Shahab L, McNeill A. Prevalence and characteristics of e-cigarette users in Great Britain: findings from a general population survey of smokers. Addict Behav. 2014;39(6):1120-1125. doi:10.1016/j.addbeh.2014.03.009

30. Adkison SE, O'Connor RJ, Bansal-Travers M, et al. Electronic nicotine delivery systems. Am J Prev Med. 2013;44(3):207-215. doi:10.1016/j.amepre.2012.10.018

31. Dockrell M, Morrison R, Bauld L, McNeill A. E-cigarettes: prevalence and attitudes in great Britain. Nicotine Tob Res. 2013;15 (10):1737-1744. doi:10.1093/ntr/ntt057

32. Douptcheva N, Gmel G, Studer J, Deline S, Etter J-F. Use of electronic cigarettes among young Swiss men: table 1. $J$ Epidemiol Community Health. 2013;67(12):1075-1076. doi:10.1136/jech-2013-203152

33. Beard E, West R, Michie S, Brown J. Association between electronic cigarette use and changes in quit attempts, success of quit attempts, use of smoking cessation pharmacotherapy, and use of stop smoking services in England: time series analysis of population trends. BMJ. 2016;i4645. doi:10.1136/bmj.i4645

34. Lee PN. Epidemiological evidence relating snus to health--an updated review based on recent publications. Harm Reduct J. 2013;10:36. doi:10.1186/1477-7517-10-36

35. McRobbie H, Bullen C, Hartmann-Boyce J, Hajek P. Electronic cigarettes for smoking cessation and reduction. In: McRobbie H, editor. Cochrane Database of Systematic Reviews. Chichester, UK: John Wiley \& Sons, Ltd; 2014.

36. Silagy C, Lancaster T, Stead L, Mant D, Fowler G. Nicotine replacement therapy for smoking cessation. In: Stead L, editor. Cochrane Database of Systematic Reviews. Chichester, UK: John Wiley \& Sons, Ltd; 2004.

37. Schick SF, Blount BC, Jacob P, et al. Biomarkers of exposure to new and emerging tobacco delivery products. Am J Physiol Lung Cell Mol Physiol. 2017;313(3):L425-52. doi:10.1152/ ajplung.00343.2016

38. Rahman MA, Hann N, Wilson A, Mnatzaganian G, WorrallCarter L. E-cigarettes and smoking cessation: evidence from a systematic review and meta-analysis. PLoS One. 2015;10(3): e0122544. doi:10.1371/journal.pone. 0122544

39. Worku D, Worku E. A narrative review evaluating the safety and efficacy of e-cigarettes as a newly marketed smoking cessation tool. SAGE Open Med. 2019;7:205031211987140. doi:10.1177/ 2050312119871405

40. Moore D, Aveyard P, Connock M, Wang D, Fry-Smith A, Barton P. Effectiveness and safety of nicotine replacement therapy assisted reduction to stop smoking: systematic review and meta-analysis. BMJ. 2009;338(apr02 3):b1024. doi:10.1136/bmj.b1024

41. Malas M, van der Tempel J, Schwartz R, et al. Electronic cigarettes for smoking cessation: a systematic review. Nicotine Tob Res. 2016;18(10):1926-1936. doi:10.1093/ntr/ntw119

42. Mersha AG, Eftekhari P, Bovill M, Tollosa DN, Gould GS. Evaluating level of adherence to nicotine replacement therapy and its impact on smoking cessation: a protocol for systematic review and meta-analysis. BMJ Open. 2020;10(9):e039775. doi:10.1136/bmjopen-2020-039775

43. Wang W, Cai Y, He Z, Feng N. Electronic cigarette use in China: awareness, prevalence and regulation. Tob Induc Dis. 2019;17. doi: $10.18332 / \mathrm{tid} / 105393$

44. Moher D, Liberati A, Tetzlaff J, Altman DG, Group TP. Preferred reporting items for systematic reviews and meta-analyses: the PRISMA statement. PLoS Med. 2009;6(7):e1000097. doi:10.1371/ journal.pmed.1000097
45. World Health Organization. WHO | Heat-Not-Burn tobacco products information sheet [Internet]. World Health Organization; 2020 [cited October 21, 2020]. Available from: https://apps.who. int/tobacco/publications/prod_regulation/heat-not-burn-productsinformation-sheet/en/index.html. Accessed July 6, 2021.

46. U.S Food and Drug Administration. Vaporizers, E-cigarettes, and other electronic nicotine delivery systems (ENDS) | FDA [Internet]. FDA; 2020 [cited October 21, 2020]. Available from: https://www.fda.gov/tobacco-products/products-ingredientscomponents/vaporizers-e-cigarettes-and-other-electronic-nicotinedelivery-systems-ends. Accessed July 6, 2021.

47. U.S Food and Drug Administration. Smokeless tobacco products, including dip, snuff, snus, and chewing tobacco | FDA [Internet]. FDA; 2020 [cited October 21, 2020]. Available from: https:// www.fda.gov/tobacco-products/products-ingredients-components /smokeless-tobacco-products-including-dip-snuff-snus-andchewing-tobacco. Accessed July 6, 2021.

48. Wadgave U, Nagesh L. Nicotine replacement therapy: an overview. Int J Health Sci. 2016;10:425-435.

49. Molyneux A. Nicotine replacement therapy. $\mathrm{Br}$ Med $\mathrm{J}$. 2004;328:454-456. doi:10.1136/bmj.328.7437.454

50. Sterne JAC, Savović J, Page MJ, et al. RoB 2: a revised tool for assessing risk of bias in randomised trials. BMJ. 2019;28:366.

51. Higgins JPT, Savović J, Page MJ; on behalf of the RoB2 Development Group. Risk of bias tools - current version of RoB 2 [Internet]. 2019 [cited October 22, 2020]. Available from: https://www.riskofbias.info/welcome/rob-2-0-tool/current-version -of-rob-2. Accessed July 6, 2021.

52. Sterne JA, Hernán MA, Reeves BC, et al. ROBINS-I: a tool for assessing risk of bias in non-randomised studies of interventions. BMJ. 2016;12:355.

53. Sterne JAC, Higgins JPT, Elbers RG, Reeves BC; the development group for R-I. Risk of bias tools - ROBINS-I detailed guidance [Internet]. 2016 [cited October 21, 2020]. Available from: https:// www.riskofbias.info/welcome/home/current-version-of-robins-i/ robins-i-detailed-guidance-2016. Accessed July 6, 2021.

54. Joksić G, Spasojević-Tišma V, Antić R, Nilsson R, Rutqvist LE. Randomized, placebo-controlled, double-blind trial of Swedish snus for smoking reduction and cessation. Harm Reduct $J$. 2011;13(8):25. doi:10.1186/1477-7517-8-25

55. Bullen $\mathrm{C}$, Howe $\mathrm{C}$, Laugesen $\mathrm{M}$, et al. Electronic cigarettes for smoking cessation: a randomised controlled trial. Lancet. 2013;382 (9905):1629-1637. doi:10.1016/S0140-6736(13)61842-5

56. Caponnetto P, Campagna D, Cibella F, et al. EffiCiency and safety of an eLectronic cigAreTte (ECLAT) as tobacco cigarettes substitute: a prospective 12-month randomized control design study. PLoS One. 2013;8(6):e66317. doi:10.1371/journal.pone. 0066317

57. Adriaens K, Van Gucht D, Declerck P, Baeyens F. Effectiveness of the electronic cigarette: an eight-week flemish study with six-month follow-up on smoking reduction, craving and experienced benefits and complaints. Int J Environ Res Public Health. 2014;11 (11):11220-11248. doi:10.3390/ijerph111111220

58. D'Ruiz CD, Graff DW, Yan XS, D'Ruiz CD, Graff DW, Yan XS. Nicotine delivery, tolerability and reduction of smoking urge in smokers following short-term use of one brand of electronic cigarettes. BMC Public Health. 2015;15:991. doi:10.1186/ s12889-015-2349-2

59. Cibella F, Campagna D, Caponnetto P, et al. Lung function and respiratory symptoms in a randomized smoking cessation trial of electronic cigarettes. Clin Sci. 2016;130(21):1929-1937. doi:10.1042/CS20160268

60. Cravo AS, Bush J, Sharma G, et al. A randomised, parallel group study to evaluate the safety profile of an electronic vapour product over 12 weeks. Regul Toxicol Pharmacol. 2016;81(Suppl 1):S1S14. doi:10.1016/j.yrtph.2016.10.003 
61. Farsalinos K, Cibella F, Caponnetto P, et al. Effect of continuous smoking reduction and abstinence on blood pressure and heart rate in smokers switching to electronic cigarettes. Intern Emerg Med. 2016;11:85-94. doi:10.1007/s11739-015-1361-y

62. Kumral TL, Saltürk Z, Yildirim G, et al. How does electronic cigarette smoking affect sinonasal symptoms and nasal mucociliary clearance? B-ENT. 2016;12:17-21.

63. Tseng T-Y, Ostroff JS, Campo A. A randomized trial comparing the effect of nicotine versus placebo electronic cigarettes on smoking reduction among young adult smokers. Nicotine Tob Res. 2016;18(10):1937-1943. doi:10.1093/ntr/ntw017

64. Walele T, Sharma G, Savioz R, Martin C, Williams J. A randomised, crossover study on an electronic vapour product, a nicotine inhalator and a conventional cigarette. Part B: safety and subjective effects. Regul Toxicol Pharmacol. 2016;74:193-199. doi:10.1016/j.yrtph.2015.12.004

65. D'Ruiz CD, O'Connell G, Graff DW, Yan XS. Measurement of cardiovascular and pulmonary function endpoints and other physiological effects following partial or complete substitution of cigarettes with electronic cigarettes in adult smokers. Regul Toxicol Pharmacol. 2017;87:36-53. doi:10.1016/j.yrtph.2017. 05.002

66. Baldassarri SR, Bernstein SL, Chupp GL, Slade MD, Fucito LM, Toll BA. Electronic cigarettes for adults with tobacco dependence enrolled in a tobacco treatment program: a pilot study. Addict Behav. 2018;80:1-5. doi:10.1016/j.addbeh.2017.11.033

67. George J, Hussain M, Vadiveloo T, et al. Cardiovascular effects of switching from tobacco cigarettes to electronic cigarettes. $J \mathrm{Am}$ Coll Cardiol. 2019;74:3112-3120. doi:10.1016/j.jacc.2019. 09.067

68. Veldheer S, Yingst J, Midya V. Pulmonary and other health effects of electronic cigarette use among adult smokers participating in a randomized controlled smoking reduction trial. Addict Behav. 2019;91:95-101. doi:10.1016/j.addbeh.2018.10.041

69. Walker N, Parag V, Verbiest M, Laking G, Laugesen M, Bullen C. Nicotine patches used in combination with e-cigarettes (with and without nicotine) for smoking cessation: a pragmatic, randomised trial. Lancet Respirat Med. 2020;8:54-64. doi:10.1016/S22132600(19)30269-3

70. Al-Delaimy WK, Myers MG, Leas EC, Strong DR, Hofstetter CR. E-cigarette use in the past and quitting behavior in the future: a population-based study. Am J Public Health. 2015;105(6):1213-1219. doi:10.2105/AJPH.2014.302482

71. Sweet L, Brasky TM, Cooper S, et al. Quitting behaviors among dual cigarette and E-cigarette users and cigarette smokers enrolled in the tobacco user adult cohort. Nicotine Tob Res. 2019;21 (3):278-284. doi:10.1093/ntr/nty222

72. Gomajee R, El-Khoury F, Goldberg M. Association between electronic cigarette use and smoking reduction in France. JAMA Intern Med. 2019;179(9):1193-1200. doi:10.1001/jamainternmed. 2019.1483

73. Flacco ME, Ferrante M, Fiore M. Cohort study of electronic cigarette use: safety and effectiveness after 4 years of follow-up. Eur Rev Med Pharmacol Sci. 2019;23(1):402-412. doi:10.26355/ eurrev_201901_16789

74. Berry KM, Reynolds LM, Collins JM, et al. E-cigarette initiation and associated changes in smoking cessation and reduction: the population assessment of tobacco and health study, 2013-2015. Tob Control. 2019;28(1):42-49. doi:10.1136/tobaccocontrol2017-054108

75. Weaver SR, Huang J, Pechacek TF, Heath JW, Ashley DL, Eriksen MP. Are electronic nicotine delivery systems helping cigarette smokers quit? Evidence from a prospective cohort study of U.S. adult smokers, 2015-2016. PLoS One. 2018;13 (7):e0198047. doi:10.1371/journal.pone.0198047
76. Mohamed MHN, Rahman A, Jamshed S, Mahmood S. Effectiveness and safety of electronic cigarettes among sole and dual user vapers in Kuantan and Pekan, Malaysia: a six-month observational study. BMC Public Health. 2018;18:1-10. doi:10.1186/s12889-018-5951-2

77. Mantey DS, Cooper MR, Loukas A, Perry CL. E-cigarette use and cigarette smoking cessation among Texas college students. Am J Health Behav. 2017;41(6):750-759. doi:10.5993/ AJHB.41.6.9

78. Shi Y, Pierce JP, White M, et al. E-cigarette use and smoking reduction or cessation in the 2010/2011 TUS-CPS longitudinal cohort. BMC Public Health. 2016;16:1-9. doi:10.1186/s12889016-3770-x

79. Rahman A, Nik Mohamad M, Jamshed S. Evaluating effectiveness and safety toward electronic cigarette among Malaysian vapers: one-month observational study. Arch Pharm Pract. 2016;7(2):43-53. doi:10.4103/2045-080X.181038

80. Polosa R, Caponnetto P, Maglia M, Morjaria JB, Russo C. Success rates with nicotine personal vaporizers: a prospective 6-month pilot study of smokers not intending to quit. $B M C$ Public Health. 2014;14:1-9. doi:10.1186/1471-2458-14-1159

81. Polosa R, Morjaria JB, Caponnetto P. Effectiveness and tolerability of electronic cigarette in real-life: a 24-month prospective observational study. Intern Emerg Med. 2014;9(5):537-546. doi:10.1007/s11739-013-0977-z

82. Polosa R, Caponnetto P, Morjaria JB, et al. Effect of an electronic nicotine delivery device (e-cigarette) on smoking reduction and cessation: a prospective 6-month pilot study. BMC Public Health. 2011;11:786. doi:10.1186/1471-2458-11-786

83. Haziza C, de La Bourdonnaye G, Skiada D, et al. Evaluation of the tobacco heating system 2.2. Part 8: 5-day randomized reduced exposure clinical study in Poland. Regul Toxicol Pharmacol. 2016;81(Suppl 2):S139-S150. doi:10.1016/j.yrtph.2016.11.003

84. Fagerstrom K, Rutqvist LE, Hughes JR. Snus as a smoking cessation aid: a randomized placebo-controlled trial. Nicotine Tob Res. 2012;14:306-312. doi:10.1093/ntr/ntr214

85. Burris JL, Carpenter MJ, Wahlquist AE, Cummings KM, Gray KM. Brief, instructional smokeless tobacco use among cigarette smokers who do not intend to quit: a pilot randomized clinical trial. Nicotine Tob Res. 2014;16(4):397-405. doi:10.1093/ ntr/ntt161

86. Allen A, Vogel RI, Meier E, et al. Gender differences in snus versus nicotine gum for cigarette avoidance among a sample of US smokers. Drug Alcohol Depend. 2016;168:8-12. doi:10.1016/ j.drugalcdep.2016.08.624

87. Carpenter MJ, Gray KM. A pilot randomized study of smokeless tobacco use among smokers not interested in quitting: changes in smoking behavior and readiness to quit. Nicotine Tob Res. 2010;12(2):136-143. doi:10.1093/ntr/ntp186

88. Carpenter MJ, Hughes JR, Gray KM, Wahlquist AE, Saladin ME, Alberg AJ. Nicotine therapy sampling to induce quit attempts among smokers unmotivated to quit: a randomized clinical trial. Arch Intern Med. 2011;171(21):1901-1907. doi:10.1001/ archinternmed.2011.492

89. Tønnesen P, Lauri H, Perfekt R, Mann K, Batra A. Efficacy of a nicotine mouth spray in smoking cessation: a randomised, double-blind trial. Eur Respir J. 2012;40(3):548-554. doi:10.1183/09031936.00155811

90. Buller DB, Halperin A, Severson HH, et al. Effect of nicotine replacement therapy on quitting by young adults in a trial comparing cessation services. J Public Health Manag Pract. 2014;20 (2):E7-E15. doi:10.1097/PHH.0b013e3182a0b8c7

91. Schnoll RA, Goelz PM, Veluz-Wilkins A, et al. Long-term nicotine replacement therapy: a randomized clinical trial. JAMA Intern Med. 2015;175(4):504-511. doi:10.1001/jamainternmed. 2014.8313 
92. Caldwell BO, Crane J. Combination nicotine metered dose inhaler and nicotine patch for smoking cessation: a randomized controlled trial. Nicotine Tob Res. 2016;18:1944-1951. doi:10.1093/ntr/ntw093

93. Schlam TR, Cook JW, Baker TB, et al. Can we increase smokers' adherence to nicotine replacement therapy and does this help them quit? Psychopharmacology. 2018;235(7):2065-2075. doi:10.1007/s00213-018-4903-y

94. The Preloading Investigators. Effects on abstinence of nicotine patch treatment before quitting smoking: parallel, two arm, pragmatic randomised trial. BMJ. 2018;361:k2164.

95. Leung MKW, Bai D, Yip BHK, et al. Combined nicotine patch with gum versus nicotine patch alone in smoking cessation in Hong Kong primary care clinics: a randomised controlled trial. BMC Public Health. 2019;19. doi:10.1186/s12889-019-7634-Z

96. Li J, Hajek P, Pesola F, et al. Cost-effectiveness of e-cigarettes compared with nicotine replacement therapy in stop smoking services in England (TEC study): a randomized controlled trial. Addiction. 2020;115(3):507-517. doi:10.1111/add.14829

97. Lechasseur A, Altmejd S, Turgeon N, et al. Variations in coil temperature/power and e-liquid constituents change size and lung deposition of particles emitted by an electronic cigarette. Physiol Rep. 2019;7(10):e14093. doi:10.14814/phy2.14093

98. Bekki K, Uchiyama S, Ohta K, Inaba Y, Nakagome H, Kunugita N. Carbonyl compounds generated from electronic cigarettes. Int $J$ Environ Res Public Health. 2014;11 (11):11192-11200. doi:10.3390/ijerph111111192

99. Bertholon JF, Becquemin MH, Annesi-Maesano I, Dautzenberg B. E-mail electronic cigarettes: a short review. Respiration. 2013;86:433-438. doi:10.1159/000353253

100. Margham J, Mcadam K, Forster M, et al. Chemical composition of aerosol from an e-cigarette: a quantitative comparison with cigarette smoke. Chem Res Toxicol. 2016;29:1662-1678. doi:10.1021/acs.chemrestox.6b00188.

101. Erythropel HC, Jabba SV, Dewinter TM, et al. Formation of flavorant-propylene glycol adducts with novel toxicological properties in chemically unstable e-cigarette liquids. Nicotine Tob Res. 2019;21(9):1248-1258. doi:10.1093/ntr/nty192

102. National Academies of Sciences Engineering and Medicine. Public Health Consequences of E-Cigarettes. Eaton D, Kwan L, Stratton K, editors. Washington (DC): National Academies Press; 2018.

103. Barufaldi LA, Guerra RL, de Cássia de Albuquerque RR, et al. Risk of smoking relapse with the use of electronic cigarettes: a systematic review with meta-analysis of longitudinal studies. Tob Prev Cessation. 2021;29:29. doi:10.18332/tpc/132964

104. Overbeek DL, Kass AP, Chiel LE, Boyer EW, Casey AMH. A review of toxic effects of electronic cigarettes/vaping in adolescents and young adults. Crit Rev Toxicol. 2020;50(6):531-538. doi:10.1080/10408444.2020.1794443

105. Fadus MC, Smith TT, Squeglia LM. The rise of e-cigarettes, pod mod devices, and JUUL among youth: factors influencing use, health implications, and downstream effects. Drug Alcohol Depend. 2019;201:85-93. doi:10.1016/j.drugalcdep.2019.04.011

106. Hartmann-Boyce J, Hong B, Livingstone-Banks J, Wheat H, Fanshawe TR. Additional behavioural support as an adjunct to pharmacotherapy for smoking cessation. Cochrane Database Syst Rev. 2019;2019(6):CD009670.

107. Rovina N, Nikoloutsou I, Katsani G, et al. Effectiveness of pharmacotherapy and behavioral interventions for smoking cessation in actual clinical practice. Ther Adv Respir Dis. 2009;3 (6):279-287. doi:10.1177/1753465809350653
108. Rutqvist LE, Fry JS, Lee PN. Systematic review of Swedish snus for smoking cessation based on primary subject data from randomised clinical trials. J Smok Cessat. 2013;8:33-44. doi:10.1017/ jsc. 2013.10

109. Gilljam H, Galanti MR. Role of snus (oral moist snuff) in smoking cessation and smoking reduction in Sweden. Addiction. 2003;98:1183-1189. doi:10.1046/j.1360-0443.2003.00379.x

110. Ramström LM, Foulds J. Role of snus in initiation and cessation of tobacco smoking in Sweden. Tob Control. 2006;15 (3):210-214. doi:10.1136/tc.2005.014969

111. Stenbeck M, Hagquist C, Rosén M. The association of snus and smoking behaviour: a cohort analysis of Swedish males in the 1990s. Addiction. 2009;104(9):1579-1585. doi:10.1111/j.13600443.2009.02661.x

112. Lund KE, McNeill A, Scheffels J. The use of snus for quitting smoking compared with medicinal products. Nicotine Tob Res. 2010;12(8):817-822. doi:10.1093/ntr/ntq105

113. Scheffels J, Lund KE, McNeill A. Contrasting snus and NRT as methods to quit smoking. An observational study. Harm Reduct J. 2012;9(1):10. doi:10.1186/1477-7517-9-10

114. Holm H, Jarvis MJ, Russell MAH, Feyerabend C. Nicotine intake and dependence in Swedish snuff takers. Psychopharmacology. 1992;108(4):507-511. doi:10.1007/BF02247429

115. Cahill K, Stevens S, Perera R, Lancaster T. Pharmacological interventions for smoking cessation: an overview and network meta-analysis. Cochrane Database Syst Rev. 2013;2013.

116. Piper ME, Smith SS, Schlam TR, et al. A randomized placebo-controlled clinical trial of 5 smoking cessation pharmacotherapies. Arch Gen Psychiatry. 2009;66 (11):1253-1262. doi:10.1001/archgenpsychiatry.2009.142

117. Sweeney CT, Fant RV, Fagerstrom KO, McGovern JF, Henningfield JE. Combination nicotine replacement therapy for smoking cessation: rationale, efficacy and tolerability. CNS Drugs. 2001;15:453-467. doi:10.2165/00023210-20011506000004

118. Lindson N, Chepkin SC, Ye W, Fanshawe TR, Bullen C, Hartmann-Boyce J. Different doses, durations and modes of delivery of nicotine replacement therapy for smoking cessation. Cochrane Database Syst Rev. 2019;2019.

119. Murray RP, Bailey WC, Daniels K, et al. Safety of nicotine polacrilex gum used by 3,094 participants in the Lung Health Study. Chest. 1996;109(2):438-445. doi:10.1378/chest.109.2.438

120. Greenland S, Satterfield MH, Lanes SF. A meta-analysis to assess the incidence of adverse effects associated with the transdermal nicotine patch. Drug Safety. 1998;18(4):297-308. doi:10.2165/ 00002018-199818040-00005

121. Woolf KJ, Zabad MN, Post JM, McNitt S, Williams GC, Bisognano JD. Effect of nicotine replacement therapy on cardiovascular outcomes after acute coronary syndromes. Am J Cardiol. 2012;110(7):968-970. doi:10.1016/j.amjcard.2012.05.028

122. Caponnetto P, Russo C, Bruno CM, Alamo A, Amaradio MD, Polosa R. Electronic cigarette: a possible substitute for cigarette dependence. Monaldi Arch Chest Dis. 2015;79(1). doi:10.4081/ monaldi.2013.104

123. Franck C, Filion KB, Kimmelman J, Grad R, Eisenberg MJ. Ethical considerations of e-cigarette use for tobacco harm reduction. Respir Res. 2016;17:1-9. doi:10.1186/s12931-016-0370-3 


\section{Publish your work in this journal}

The Journal of Multidisciplinary Healthcare is an international, peerreviewed open-access journal that aims to represent and publish research in healthcare areas delivered by practitioners of different disciplines. This includes studies and reviews conducted by multidisciplinary teams as well as research which evaluates the results or conduct of such teams or healthcare processes in general. The journal covers a very wide range of areas and welcomes submissions from practitioners at all levels, from all over the world. The manuscript management system is completely online and includes a very quick and fair peer-review system. Visit http://www.dovepress.com/testimonials. php to read real quotes from published authors. 